\title{
Bridging the Gap Between Designed and Implemented Controllers via Adaptive Robust Discrete Sliding Mode Control
}

\author{
M. R. Amini*a, M. Shahbakhtia , S. Pan ${ }^{\mathrm{b}}$, J. K. Hedrick ${ }^{\mathrm{c}}$ \\ ${ }^{a}$ Department of Mechanical Engineering-Engineering Mechanics, Michigan Technological \\ University, Houghton, MI 49931 \\ ${ }^{b}$ Department of Mechanical Engineering, Stanford University, Stanford, CA 94305 \\ ${ }^{c}$ Department of Mechanical Engineering, University of California, Berkeley, CA 94720
}

\begin{abstract}
Bridging the gap between designed and implemented model-based controllers is a major challenge in the design cycle of industrial controllers. This gap is created due to (i) digital implementation of controller software that introduces sampling and quantization uncertainties, and (ii) uncertainties in the modeled plant's dynamics. In this paper, a new adaptive and robust modelbased control approach is developed based on a nonlinear discrete sliding mode controller (DSMC) formulation to mitigate implementation imprecisions and model uncertainties, that consequently minimizes the gap between designed and implemented controllers. The new control approach incorporates the predicted values of the implementation uncertainties into the controller structure. Moreover, a generic adaptation mechanism will be derived to remove the errors in the nonlinear modeled dynamics. The proposed control approach is illustrated on a nonlinear automotive engine control problem. The designed DSMC is tested in real-time in a processor-in-the-loop (PIL) setup using an actual electronic control unit (ECU). The verification test results show that the proposed controller design, under ADC and model uncertainties, can improve the tracking performance up to $60 \%$ compared to a conventional controller design.
\end{abstract}

*Corresponding author

Email addresses: mamini@mtu.edu (M. R. Amini*), mahdish@mtu.edu (M. Shahbakhti), slpan@stanford.edu (S. Pan), khedrick@me.berkeley.edu (J. K. Hedrick)

Preprint submitted to Control Engineering Practice

September 28, 2016

(C) 2016. This manuscript version is made available under the Elsevier user license http://www.elsevier.com/open-access/userlicense/1.0/ 
Keywords: Sliding Mode Control, Model Uncertainty, Implementation Imprecisions, Analog to Digital Conversion, Verification and Validation

\section{Introduction}

Verification and validation $(\mathrm{V} \& \mathrm{~V})$ is part of the design cycle of a controller to verify the controller software and validate the desired performance of the designed controller. The $\mathrm{V} \& \mathrm{~V}$ process confirms that the implemented controller (i) has the required conceptual description and specifications, and (ii) can meet the desired control targets. The traditional V\&V cycle of various electronic control unit (ECU) functions is completed with multiple software-in-the-loop (SIL), processor-in-the-loop (PIL), and hardware-in-theloop (HIL) iterations, which are complex and time consuming. Reducing the cost and time for completing the $\mathrm{V} \& \mathrm{~V}$ is a major challenge for all complex control systems. There are two main sources of uncertainties in the design cycle of industrial model-based controllers that cause errors in a V\&V cycle. First, the imprecisions arise during digital implementation of the controller upon analog-to-digital (ADC) conversion of signals at the input/output (I/O) of the controller due to sampling and quantization. Second, uncertainties exist in the modeled plant's dynamics used in the controller formulation. If these uncertainties could be identified and fixed during the early stages of the controller software design, the V\&V process would cost 10 times less $[1,2]$. Thus, designing a robust controller that tries to minimize the impact of implementation and model imprecisions can reduce the V\&V cost significantly.

A sliding mode controller (SMC) is a robust controller that can be applied to both linear and nonlinear systems, and allows for dealing with uncertainties, un-modeled dynamics, and disturbances. SMC design converts a complicated dynamic system into a first-order system through a sliding surface transformation. Previous studies in the literature (shown in Figure 1) have demonstrated the capabilities of SMC in handling implementation and model uncertainties. There are two main groups in which the SMC design with robustness to implementation imprecisions can be studied: (i) continuous-time designs, and (ii) discrete-time designs. For the first group, results in [3] showed that the robustness of the implemented controller against ADC uncertainties can be improved by incorporating the maximum ADC uncertainty bounds into the controller equations. Converting the controller design from a single-input single-output (SISO) to a multi-input multi-output (MIMO) 


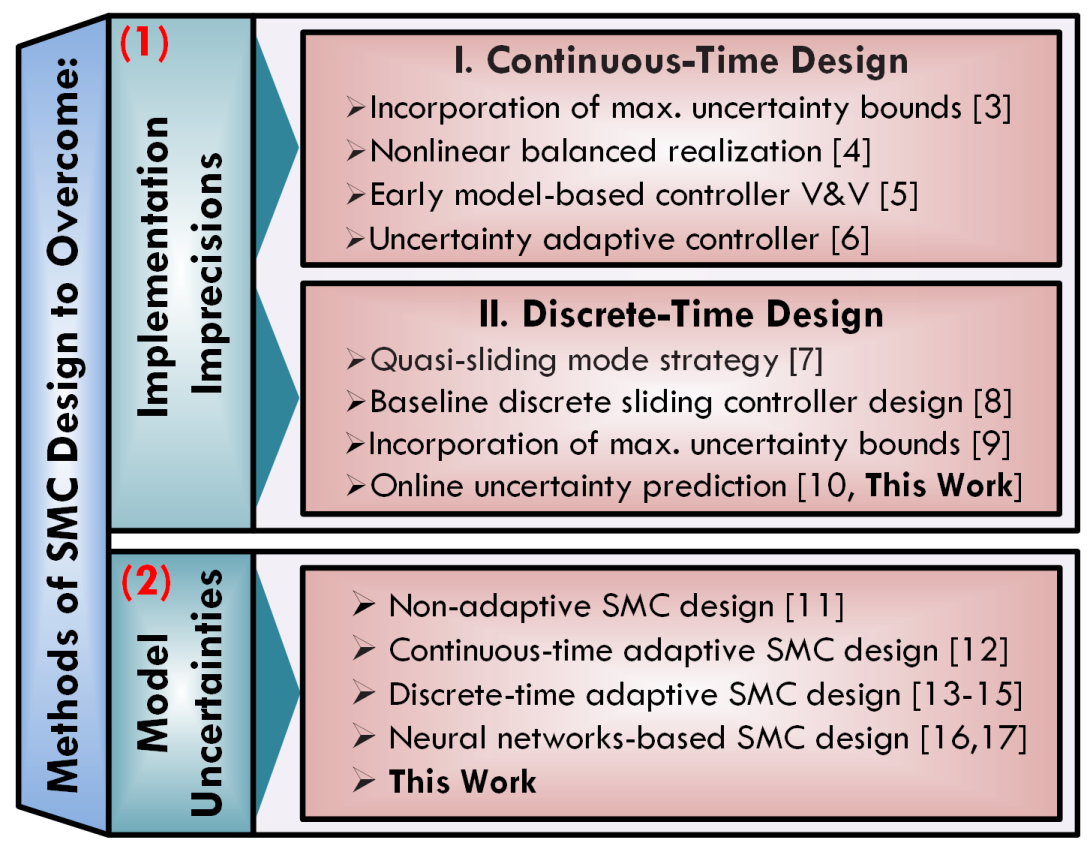

Figure 1: Background of some previous studies $[3,4,5,6,7,8,9,10,11,12,13,14,15$, $16,17]$ on SMC design against ADC and modeling uncertainties.

formulation is another effective approach to enhance controller robustness by minimizing the correlation between the sliding variables via a nonlinear balanced realization method [4]. Additionally, it is essential to examine the controller structure to find states/variables with the largest numerical noise. Then, by removing those noise sources from the controller structure, the robustness characteristics of the controller can be significantly improved [5]. The last approach is to incorporate an uncertainty adaptive mechanism into the SMC formulation by which the control actions can be modified to compensate the effect of ADC uncertainties [6].

We illustrated in our previous studies that a discrete sliding mode controller (DSMC), which explicitly includes the sampling time, shows better performance under sampling imprecisions compared to a continuous-time SMC that is digitally implemented [7]. Quasi and baseline discrete sliding mode controllers were introduced and studied under implementation imprecisions in [7] and [8], respectively. The robustness of the DSMC design was enhanced by incorporating the maximum ADC uncertainty bounds on control inputs in [9]. The results showed that the proposed DSMC with maximum ADC uncertainty bounds can lead to better tracking performance in 
comparison with the baseline SMC/DSMC. The incorporation of maximum ADC uncertainty bounds requires an ideal symbolic model of the controller which prevents the online uncertainty bounds calculation. Moreover, the results in [3] and [9] showed that the calculated maximum ADC uncertainty bounds, which are based on a worst case scenario, lead to conservative controller design and large control actions, which are not desired. To this end, in our previous work [10], we developed an online technique to predict and propagate sampling and quantization imprecisions on control signals. The results showed that not only is the conservative controller design is avoided by using the new ADC uncertainty prediction technique, but also that the controller robustness improves significantly compared to a baseline DSMC with no knowledge about the implementation uncertainties.

Handling the modeling uncertainty in SMC design has been the subject of few previous works in the literature. The model uncertainty/mismatch has been addressed using non-adaptive [11], continuous-time adaptive [12], discrete-time adaptive [13, 14, 15], and neural network-based [16, 17] SMC designs. The adaptive DSMC formulation from [18] presents a generic solution for removing a multiplicative type of model uncertainty for a general class of nonlinear systems. However, the proposed adaptive DSMC in [18] was not investigated under sampling and quantization imprecisions. Besides, regardless of the state equations of the physical model, it was assumed that an unknown multiplicative term can represent the existing uncertainty in the model. In this paper, in addition to the unknown multiplicative uncertainty term, a generic unknown additive term is considered in the model's equation to extend the adaptive DSMC formulation to a broader range of dynamical systems. This paper builds upon our previous works in Refs. [10, 19].

The contribution of this paper is twofold. First, an online uncertainty prediction and propagation technique is proposed to estimate the ADC uncertainties on a control signal based on a new DSMC formulation. Second, the new DSMC with predicted implementation imprecisions is extended to handle additive and multiplicative types of model uncertainties for nonlinear systems using a discrete Lyapunov stability analysis. The asymptotic stability of the proposed controller is proven by invoking the new extension of the LaSalle's Invariance Principle for nonlinear systems with discontinuity (New Invariance Principle). To the best of authors' knowledge, this paper is the first integrated study which aims to handle both ADC (sampling and quantization) and model (multiplicative and additive) uncertainties via a generic adaptive robust DMSC design. The performance of the proposed adaptive 
robust DSMC is evaluated for a highly nonlinear automotive engine control problem. Finally, the designed controller is experimentally verified on a real ECU.

\section{Design of Adaptive Robust DSMC}

A general class of SISO affine nonlinear systems can be described using the following state space representation:

$$
\begin{gathered}
\dot{x}=f(x(t))+g(x(t)) u(t) \\
y=h(x(t))
\end{gathered}
$$

where $x \in \mathbb{R}^{r}$ is the state vector and $u \in \mathbb{R}$ and $h \in \mathbb{R}$ denote the system input and output, respectively. $g(x)$ is the control input coefficient and $f(x(t))$ represents the dynamics of the plant and does not depend on the inputs. The tracking control problem is to drive the state of the system $(x)$ to its desired value $\left(x_{d}\right)$. To this end, a SISO DSMC is formulated. Sliding surface variable $(s)$ is defined as the difference between the desired $\left(x_{d}\right)$ and the measured signal $(x)$ at $i^{\text {th }}$ time step as follows:

$$
s(i)=x(i)-x_{d}(i)
$$

The control input $u(i)$ is obtained according to the following sliding reaching law $[8,10]$ :

$$
|s(i+1)| \leq \rho|s(i)|
$$

where $0<\rho<1$ is the tunable DSMC gain. The first order Euler approximation, $\dot{x} \approx T^{-1} \times(x(i+1)-x(i))$, is used to discretize the nonlinear system in Eq. (1):

$$
x(i+1)=x(i)+T f(i)+g(x(i)) u(i) T
$$

It is assumed that a unique control input, either physical or synthetic, exists for every single sliding surface that drives the corresponding state to the desired value in a finite time. The baseline DMSC can be formulated for each state of the discrete nonlinear system in Eq. (4) with the sliding surface function and reaching law described in Eq. (2) and (3) as follows:

$$
u(i)=\frac{1}{g T}\left[(\rho-1) x(i)-\rho x_{d}(i)-T f+x_{d}(i+1)\right]
$$


Eq. (5) calculates the control input of an ideal nonlinear controller in which no imprecisions on the inputs and outputs of the controller are considered. Moreover, it is assumed that the modeled plant's dynamic is accurate. These two assumptions are the main sources of the gap between the designed and the implemented controllers. Here, both sources of uncertainties, ADC imprecisions and errors in modeled plant's dynamic, are included in the DSMC design. Next, through the proposed robust adaptive DSMC formulation, both ADC and model uncertainties will be addressed. The uncertainties in the modeled dynamics $(f)$ can be presented using unknown multiplicative $(\beta)$ and additive $(\alpha)$ terms in the presence of hardware (ADC) imprecisions $(\delta(i))$ using the nominal discrete nonlinear model in Eq. (4):

$$
x(i+1)=x(i)+T(\beta f(i)+\alpha)+T g(x(i))(u(i)+\delta(i))
$$

The proposed control design procedure in this paper for handling ADC and model uncertainties includes two major parts: (i) the ADC imprecisions on control signal $(\delta(i))$ are compensated by predicting and incorporating the sampling and quantization uncertainties on measured signals, (ii) the multiplicative and additive unknown terms in the plant's nominal model are driven to their nominal values using an adaptation technique. Figure 2 shows the schematic of the proposed adaptive robust DSMC design in this paper which will be discussed in the following sections.

In Section 2.1, first an online ADC uncertainty prediction mechanism will be discussed to estimate sampling and quantization uncertainties on the measured signals due to ADC (Section 2.1.1). Design of a DSMC with robustness to $\mathrm{ADC}$ uncertainties requires the knowledge of the propagated ADC imprecisions on the control signal. Thus, an online ADC imprecisions propagation mechanism for nonlinear systems is developed and presented in Section 2.1.2. Next (Section 2.1.3), the estimation of the propagated ADC uncertainties on control signals is incorporated into the baseline DSMC design to compensate $\delta(i)$ term in Eq. (6). In Section 2.2, the adaptation laws for handling two types of model uncertainties (multiplicative and additive) are derived based on a Lyapunov stability argument. Using the detailed nonlinear discrete Lyapunov stability analysis, not only can the adaptation rules be concluded, but also the asymptotic stability of the closed loop system can be guaranteed. 


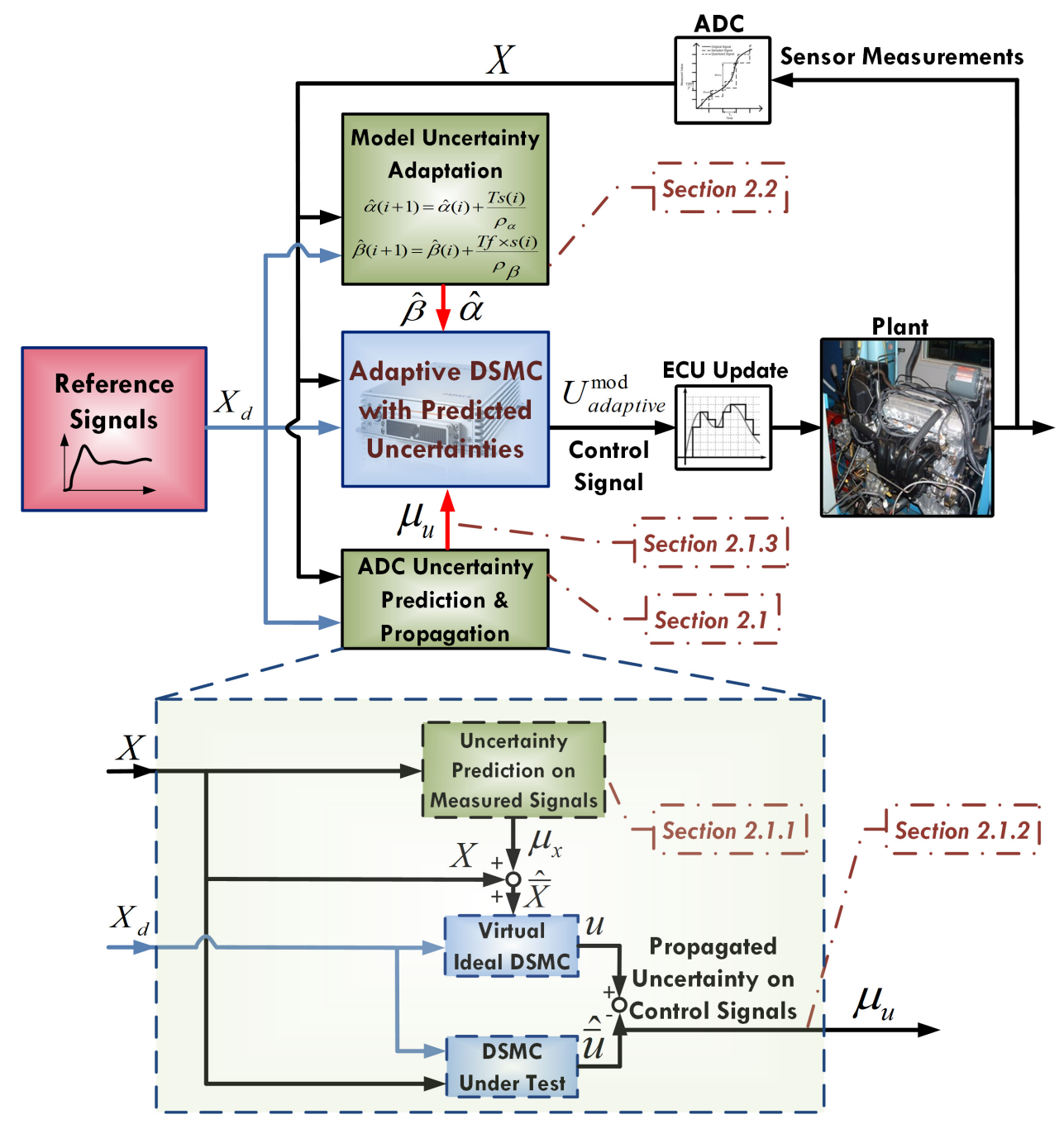

Figure 2: Schematic of the adaptive DSMC with online estimations of uncertainties on measured and control signals. $X_{d}$ is the desired trajectories (reference), $X$ represents the measured signals from the plant after ADC, $U_{\text {adaptive }}^{\text {mod }}$ is the control input, $\mu_{X}$ is the predicted ADC uncertainties on measured signals, $\mu_{U}$ is the propagated ADC uncertainties on control signals, and $\hat{\alpha}, \hat{\beta}$ are the estimated unknown parameters of the model.

\subsection{Robustness against $A D C$ Uncertainties}

\subsubsection{ADC Uncertainty Prediction}

The introduced sampling and quantization imprecisions by ADC on a measured analog signal are demonstrated in Figure 3. The signal slope and the introduced round-off error due to quantization can be used to obtain an 
estimation of the ADC uncertainty on the measured signal. The sampling uncertainty $\left(\mu_{x_{s}}(i)\right)$ on a measured signal at $i^{\text {th }}$ time step can be calculated as follows:

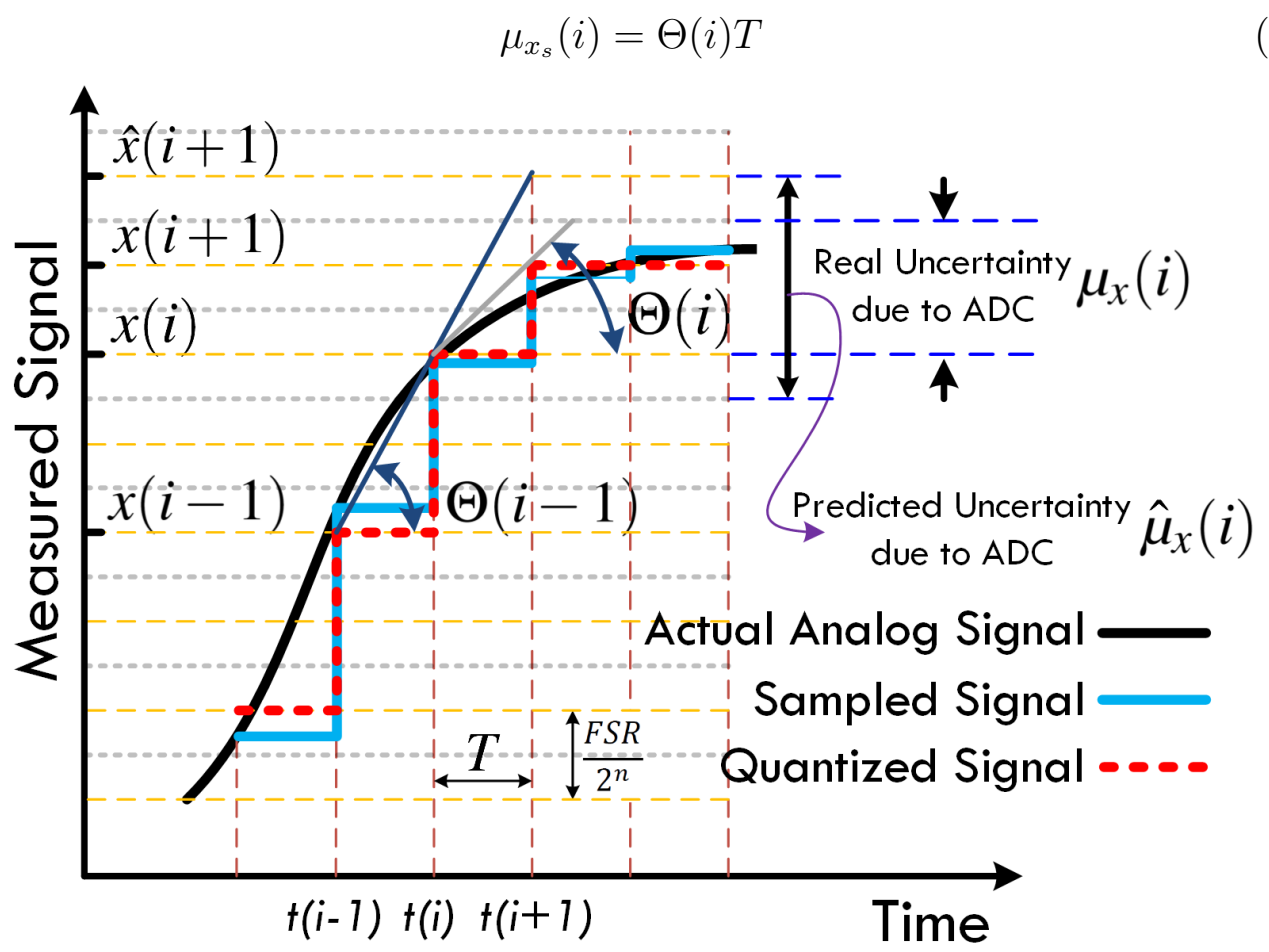

Figure 3: ADC uncertainty on a measured analog signal due to sampling and quantization. Symbols are explained in Eq. (8) to (11).

Where $T$ is the sampling time and $\Theta$ is the signal slope. The uncertainty should be estimated based on the value of the measured signal at the current time step, so that it can be applied to the next cycle. To this end, $\Theta(i)$ is calculated using forward $(f w)$ difference approximation according to the values of the sampled signal. In practice, the sampled data is then digitized at a rate equal to the $\mathrm{ADC}$ quantization level. This means that the values of the sampled signal before quantization are not available and the only information that controller has access to are values of the sampled and quantized signal after ADC. Therefore, the signal slope is calculated with respect to the sampled and quantized signal after ADC:

$$
\Theta^{f w}(i)=\frac{x(i+1)-x(i)}{T}
$$


In Eq. (8), the value of $x(i+1)$ is unknown at $i^{\text {th }}$ time step. $x(i+1)$ is predicted $(\hat{x}(i+1))$ to obtain the uncertainty due to ADC. One approach to obtain $(\hat{x}(i+1))$ is to assume that the calculated signal slope from Eq. (8) is equal to the calculated slope based on the backward $(b w)$ difference approximation $\left(\Theta^{b w}(i)=T^{-1}(x(i)-x(i-1))\right)$ :

$$
\Theta^{f w}(i)=\Theta^{b w}(i) \Rightarrow \hat{x}(i+1)=T \Theta^{b w}(i)+x(i)
$$

Moreover, the uncertainty introduced by quantization $\left(\mu_{x_{q}}(i)\right)$ can be calculated at each time step by considering a round-off error [5]:

$$
\mu_{x_{q}}(i)=\frac{1}{2} \frac{F S R}{2^{n}}
$$

where $F S R$ is the full scale range of the measured signal and $n$ is the ADC's number of bits and represents the ADC resolution. Overall, the estimated sampling and quantization uncertainties $\left(\hat{\mu}_{x}\right)$ on measured signals at $i^{\text {th }}$ time step can be obtained as follows by using Eq. (7), (9), and (10):

$$
\hat{\mu}_{x}(i)=\hat{\mu}_{x_{s}}(i)+\mu_{x_{q}}(i)=x(i)-x(i-1)+\frac{1}{2} \frac{F S R}{2^{n}}
$$

where $\hat{\mu}_{x_{s}}$ is the predicted sampling uncertainty with respect to the value of the predicted sampled signal at $i+1^{t h}$ time step $\left(\hat{x}_{i+1}\right)$. The accuracy of Eq. (11) is sensitive to the magnitude of the changes in the signal's slope. Moreover, the accuracy of the uncertainty estimation depends on the ADC quantization level. Lower ADC resolution leads to poor uncertainty estimation. During the time interval between two time steps, the output of a controller is constant. Thus the predicted value for ADC imprecisions at each time step can be assumed to be constant over the next time interval. In other words, Eq. (11) determines ADC uncertainty prediction at each time step until the next time step, since during the time interval between two time steps, the output of a controller is assumed to be constant.

\subsubsection{ADC Uncertainty Propagation on Control signal}

In the next step, it is required to obtain the propagated implementation imprecisions on control signals, which will be incorporated into the baseline DSMC formulation (Eq. (5)) to enhance the robustness characteristics against sampling and quantization imprecisions. For nonlinear systems, unlike the linear systems, it is not possible to conduct a real-time and analytic 
noise propagation to obtain the uncertainty bounds on control signals [10]. Here an online mechanism is proposed to estimate the propagated ADC uncertainties on control signals. The uncertainty prediction and propagation mechanism contains two auxiliary baseline DSMCs, including a virtual ideal DSMC and the DSMC under test shown in Figure 2. Desired trajectories for both auxiliary DSMCs are the same. The feedback signal to the DSMC under test is a vector of the measured signals after $\operatorname{ADC}(\mathbf{X})$, while the input to the virtual ideal DSMC is the estimated vector of the measured signals before $\operatorname{ADC}(\hat{\overline{\mathbf{X}}})$. $\hat{\overline{\mathbf{X}}}$ is estimated according to the real values of the measured signals after ADC and the predicted ADC uncertainties on measured signals $\left(\mu_{X}=\operatorname{diag}\left[\mu_{x_{1}}, \mu_{x_{2}}, \ldots, \mu_{x_{r}}\right]\right)$ from Eq. (11):

$$
\hat{\overline{\mathbf{X}}}=\mu_{X}+\mathbf{X}
$$

It is assumed that by using Eq. (12), the ADC uncertainty on measured signal is compensated. This means that the only difference between the DSMC under test and the virtual ideal DSMC is the ADC uncertainties on measured signals. Therefore, the propagated uncertainty vector $\left(\mu_{U}\right)$ on control signals can be obtained according to the difference between the control signals of these two controllers:

$$
\mu_{U}=\operatorname{diag}\left[\hat{\bar{u}}_{1}-u_{1}, \ldots, \hat{\bar{u}}_{r}-u_{r}\right]
$$

where $\hat{\bar{u}}_{1, \ldots, r}$ are the calculated control inputs from the ideal DSMC and $u_{1, \ldots, r}$ are the control inputs from the DSMC under test.

\subsubsection{Inclusion of $A D C$ Uncertainties into the $D S M C$}

Structure of the baseline DSMC (Eq. (5)) is modified against ADC imprecisions by incorporating the estimated uncertainties on control signals $(\hat{\delta}(i))$ into the DSMC formulation:

$$
u^{\bmod }(i)=u(i)-\hat{\delta}(i)
$$

where $u^{\text {mod }}$ is the modified controller input and $u$ is calculated with respect to Eq. (5). $\hat{\delta}(i)$ is obtained according to the predicted uncertainties on control signals $\left(\mu_{u}(i)\right)$, and it is assumed to be able to compensate for the ADC imprecisions on control signal $(\delta(i))$ :

$$
\hat{\delta}(i)=\mu_{u}(i) s(i)
$$


In the absence of model uncertainties, by inclusion of propagated ADC uncertainties on control signals $\left(\mu_{u}\right)$, the baseline DSMC for a nonlinear system in Eq. (4) is modified as follows:

$$
u^{m o d}(i)=\frac{1}{g T}\left[(\rho-1) x(i)-\rho x_{d}(i)-f T+x_{d}(i+1)\right]-\mu_{u}(i) s(i)
$$

The reason for using the continuous term $s(i)$ in Eq. (15) instead of the traditional switching signum function, $\operatorname{sgn}(s(i))$, is to avoid chattering phenomena which occurs in discrete systems during implementation of the signum function $[9,12]$. In other words, the modified DSMC formulation in Eq. (16) provides a "smoother version" of traditional sliding mode controller design. The proposed "smoothed" sliding model controller drives the trajectories to the boundary layer around the sliding manifold in finite time which gives guaranteed minimum tracking error under ADC uncertainties.

\subsection{Adaptation Against Model Uncertainties}

The uncertainties in the modeled dynamics $(f)$ can be expressed using unknown multiplicative $(\beta)$ and additive $(\alpha)$ terms in the presence of hardware $(\mathrm{ADC})$ imprecisions $(\delta(i))$ by using the modified control input $\left(u^{\bmod }(i)\right)$ from Eq. (14) in the discrete nonlinear model in Eq. (6):

$$
x(i+1)=x(i)+T(\beta f+\alpha)+T g(i)\left(u^{\bmod }(i)+\delta(i)\right)
$$

Here, an adaptive DSMC theory is developed for handling the multiplicative and additive types of model uncertainties using Lyapunov stability theory. By applying the sliding reaching law in Eq. (3) for the discrete nonlinear system in Eq. (17) with uncertainty terms $(\alpha$ and $\beta$ ), the following relationship is concluded:

$$
s(i+1)=x(i)+T(\beta f+\alpha)+T g(i)\left(u^{\bmod }(i)+\delta(i)\right)-x_{d}(i+1)
$$

where $\alpha$ and $\beta$ are unknown and constant. Modified control input $\left(u^{\bmod }\right)$ is calculated according to Eq. (16) in the presence of the uncertainty terms:

$u^{\bmod }(i)=\frac{1}{g T}\left[(\rho-1) x(i)-\rho x_{d}(i)-(\hat{\beta}(i) f+\hat{\alpha}(i)) T+x_{d}(i+1)\right]-\mu_{u}(i) s(i)$ 
where $\hat{\beta}(i)$ and $\hat{\alpha}(i)$ are estimations of the unknown multiplicative and additive parameters, respectively.

We assume that by incorporating the predicted ADC function $\left(\mu_{u}(i) s(i)\right)$ into the DSMC structure, the ADC imprecisions on control signal $(\delta(i))$ is compensated. By substituting Eq. (19) into Eq. (18), the sliding surface dynamics can be simplified as follows:

$$
s(i+1)=\rho s(i)+T((\beta-\hat{\beta}(i)) f+(\alpha-\hat{\alpha}(i)))
$$

Two new terms $(\tilde{\alpha}$ and $\tilde{\beta})$ are defined to represent the difference between the unknown and estimated uncertainty terms, $\tilde{\alpha}(i)=\alpha-\hat{\alpha}(i)$ and $\tilde{\beta}(i)=$ $\beta-\hat{\beta}(i)$. The sliding surface dynamics, in terms of the DSMC gain $(\rho)$ and the errors in estimating the unknown parameters, can be obtained after substituting $\tilde{\alpha}$ and $\tilde{\beta}$ into Eq. (20):

$$
s(i+1)=\rho s(i)+T(\tilde{\beta}(i) f+\tilde{\alpha}(i))
$$

A Lyapunov-based analysis is employed to (i) determine the stability of the closed-loop system, and (ii) derive the adaptation laws. The following Lyapunov function candidate is proposed:

$$
V(i)=\frac{1}{2} s^{2}(i)+\frac{1}{2} \rho_{\beta} \tilde{\beta}^{2}(i)+\frac{1}{2} \rho_{\alpha} \tilde{\alpha}^{2}(i)
$$

where $\rho_{\beta}>0$ and $\rho_{\alpha}>0$ are tunable adaptation gains that are chosen for the numerical sensitivity of the unknown multiplicative and additive parameter estimations, respectively. The proposed Lyapunov function is positive definite and quadratic with respect to the sliding variable $(s), \tilde{\beta}$, and $\tilde{\alpha}$. Similar to continuous-time systems, in which the negative definite condition is required for the derivative of the Lyapunov function to guarantee the asymptotic stability, in the discrete time domain, investigation of a Lyapunov difference function is required for the Lyapunov stability analysis. The Lyapunov difference function is calculated using an implicit approach in which the value of the Lyapunov function at the subsequent time step is obtained using a three-variable Taylor series expansion: 


$$
\begin{gathered}
V(i+1)=V(i)+ \\
\frac{\partial V(i)}{\partial s(i)} \Delta s(i)+\frac{\partial V(i)}{\partial \tilde{\beta}(i)} \Delta \tilde{\beta}(i)+\frac{\partial V(i)}{\partial \tilde{\alpha}(i)} \Delta \tilde{\alpha}(i) \\
+\frac{1}{2} \frac{\partial^{2} V(i)}{\partial s^{2}(i)} \Delta s^{2}(i)+\frac{1}{2} \frac{\partial^{2} V(i)}{\partial \tilde{\beta}^{2}(i)} \Delta \tilde{\beta}^{2}(i)+\frac{1}{2} \frac{\partial^{2} V(i)}{\partial \tilde{\alpha}^{2}(i)} \Delta \tilde{\alpha}^{2}(i) \\
+\frac{\partial^{2} V(i)}{\partial s(i) \partial \tilde{\beta}(i)} \Delta s(i) \times \Delta \tilde{\beta}(i)+\frac{\partial^{2} V(i)}{\partial s(i) \partial \tilde{\alpha}(i)} \Delta s(i) \times \Delta \tilde{\alpha}(i) \\
+\frac{\partial^{2} V(i)}{\partial \tilde{\beta}(i) \partial \tilde{\alpha}(i)} \Delta \tilde{\beta}(i) \times \Delta \tilde{\alpha}(i)+\ldots
\end{gathered}
$$

where:

$$
\begin{array}{r}
\Delta s(i) \equiv s(i+1)-s(i) \\
\Delta \tilde{\beta}(i) \equiv \tilde{\beta}(i+1)-\tilde{\beta}(i) \\
\Delta \tilde{\alpha}(i) \equiv \tilde{\alpha}(i+1)-\tilde{\alpha}(i)
\end{array}
$$

and the partial derivatives are as follows:

$$
\begin{gathered}
\frac{\partial V(i)}{\partial s(i)}=s(i), \quad \frac{\partial V(i)}{\partial \tilde{\beta}(i)}=\rho_{\beta} \tilde{\beta}(i), \quad \frac{\partial V(i)}{\partial \tilde{\alpha}(i)}=\rho_{\alpha} \tilde{\alpha}(i) \\
\frac{\partial^{2} V(i)}{\partial s^{2}(i)}=1, \quad \frac{\partial^{2} V(i)}{\partial \tilde{\beta}^{2}(i)}=\rho_{\beta}, \quad \frac{\partial^{2} V(i)}{\partial \tilde{\alpha}^{2}(i)}=\rho_{\alpha}, \\
\frac{\partial^{2} V(i)}{\partial s(i) \partial \tilde{\beta}(i)}=0, \quad \frac{\partial^{2} V(i)}{\partial s(i) \partial \tilde{\alpha}(i)}=0, \quad \frac{\partial^{2} V(i)}{\partial \tilde{\beta}(i) \partial \tilde{\alpha}(i)}=0
\end{gathered}
$$

Next, the Lyapunov difference function $(\Delta V(i)=V(i+1)-V(i))$ is calculated by substituting Eq. (25) into Eq. (23):

$$
\begin{gathered}
\Delta V(i)=s(i) \Delta s(i)+\rho_{\beta} \tilde{\beta}(i) \Delta \tilde{\beta}(i)+\rho_{\alpha} \tilde{\alpha}(i) \Delta \tilde{\alpha}(i) \\
+\frac{1}{2} \Delta s^{2}(i)+\frac{1}{2} \rho_{\beta} \Delta \tilde{\beta}^{2}(i)+\frac{1}{2} \rho_{\alpha} \Delta \tilde{\alpha}^{2}(i)+\ldots
\end{gathered}
$$

As can be observed from Eq. (25), all higher order ( $>2)$ derivatives are zero. After substitution of Eq. (24) and (25) into Eq. (26), the Lyapunov difference 
function is simplified as follows:

$$
\begin{aligned}
& \Delta V(i) \approx s(i)(s(i+1)-s(i)) \\
& +\rho_{\beta} \tilde{\beta}(i)(\tilde{\beta}(i+1)-\tilde{\beta}(i))+\rho_{\alpha} \tilde{\alpha}(i)(\tilde{\alpha}(i+1)-\tilde{\alpha}(i)) \\
& \quad+\frac{1}{2} \Delta s^{2}(i)+\frac{1}{2} \rho_{\beta} \Delta \tilde{\beta}^{2}(i)+\frac{1}{2} \rho_{\alpha} \Delta \tilde{\alpha}^{2}(i)
\end{aligned}
$$

which yields:

$$
\begin{gathered}
\Delta V(i) \approx(\rho-1) s^{2}(i)+T \tilde{\beta}(i) f s(i)+T \tilde{\alpha}(i) s(i)+ \\
+\rho_{\beta} \tilde{\beta}(i)(\tilde{\beta}(i+1)-\tilde{\beta}(i))+\rho_{\alpha} \tilde{\alpha}(i)(\tilde{\alpha}(i+1)-\tilde{\alpha}(i)) \\
+\frac{1}{2} \Delta s^{2}(i)+\frac{1}{2} \rho_{\beta} \Delta \tilde{\beta}^{2}(i)+\frac{1}{2} \rho_{\alpha} \Delta \tilde{\alpha}^{2}(i)
\end{gathered}
$$

and finally:

$$
\begin{gathered}
\Delta V(i) \approx-(1-\rho) s^{2}(i) \\
+\rho_{\alpha} \tilde{\alpha}(i)\left(\tilde{\alpha}(i+1)-\tilde{\alpha}(i)+\frac{T s(i)}{\rho_{\alpha}}\right) \\
+\rho_{\beta} \tilde{\beta}(i)\left(\tilde{\beta}(i+1)-\tilde{\beta}(i)+\frac{T f s(i)}{\rho_{\beta}}\right) \\
+\frac{1}{2}\left(\Delta s^{2}(i)+\rho_{\beta} \Delta \tilde{\beta}^{2}(i)+\rho_{\alpha} \Delta \tilde{\alpha}^{2}(i)\right)
\end{gathered}
$$

The following adaptation laws are chosen to update the errors in estimating the unknown multiplicative $(\tilde{\beta})$ and additive $(\tilde{\alpha})$ parameters:

$$
\begin{gathered}
\tilde{\beta}(i+1)=\tilde{\beta}(i)-\frac{T f s(i)}{\rho_{\beta}} \\
\tilde{\alpha}(i+1)=\tilde{\alpha}(i)-\frac{T s(i)}{\rho_{\alpha}}
\end{gathered}
$$

Upon incorporating the adaptation laws from Eq. (30) in the Lyapunov difference function, we have:

$$
\begin{gathered}
\Delta V(i) \approx-(1-\rho) s^{2}(i) \\
+\frac{1}{2}\left(\Delta s^{2}(i)+\rho_{\beta} \Delta \tilde{\beta}^{2}(i)+\rho_{\alpha} \Delta \tilde{\alpha}^{2}(i)\right)
\end{gathered}
$$


Next the second order terms in Eq. (31) are expanded and $\Delta \tilde{\beta}$ and $\Delta \tilde{\alpha}$ are replaced by the adaptation equations from Eq. (30):

$$
\begin{gathered}
\Delta V(i) \approx-(1-\rho) s^{2}(i) \\
+\frac{1}{2}\left((s(i+1)-s(i))^{2}+\rho_{\beta}\left(-\frac{T f s(i)}{\rho_{\beta}}\right)^{2}+\rho_{\alpha}\left(-\frac{T s(i)}{\rho_{\alpha}}\right)^{2}\right)
\end{gathered}
$$

For small enough $T$, all the terms in Eq. (32) with $T^{2}$ can be neglected. Additionally, $s(i+1)$ is replaced by $\rho s(i)$ :

$$
\Delta V(i) \approx\left((\rho-1)+\frac{1}{2}(\rho-1)^{2}\right) s^{2}(i)
$$

which yields:

$$
\Delta V(i) \approx \frac{1}{2}\left(\rho^{2}-1\right) s^{2}(i)
$$

Since $1>\rho>0$, Eq. (34) concludes that there exists a region around $s=0$, $\tilde{\beta}=0$ and $\tilde{\alpha}=0$ where the Lyapunov difference function is negative semidefinite. This means that the sliding variable (the tracking error, $s$ ) converges to zero, and the errors in estimating the unknown parameters $(\tilde{\beta}, \tilde{\alpha})$ are at least bounded [20].

In order to guarantee the global asymptotic stability characteristic of the proposed adaptive robust DMSC it is required to show that all the higher order Lyapunov differences vanish [15]. To this end, the analysis begins with the second Lyapunov difference function $(\Delta V(i+1)-\Delta V(i))$ :

$$
\begin{gathered}
\Delta V(i+1)-\Delta V(i) \approx \frac{1}{2}\left(\rho^{2}-1\right) s^{2}(i+1)-\frac{1}{2}\left(\rho^{2}-1\right) s^{2}(i) \\
\approx \frac{1}{2}\left(\rho^{2}-1\right)\left(s^{2}(i+1)-s^{2}(i)\right)
\end{gathered}
$$

where $\Delta V(i+1)$ is calculated from Eq. (34) at $i+1^{\text {th }}$ time step. The objective is to show that $\Delta V(i+1)-\Delta V(i)=0$ when $s(i)=0$. It can be observed from the right hand side of Eq. (35) that $\Delta V(i+1)-\Delta V(i)=0$ if $s^{2}(i+1)-s^{2}(i)=0$, which is satisfied according to either of the following conditions:

$$
\begin{array}{cc}
s(i+1)=s(i)=0 & (I) \\
s(i+1)=s(i) \neq 0 & (I I)
\end{array}
$$


According to Eq. (34), if $s(i)=0, \Delta V(i)=0$. On the other hand, assuming that the unknown uncertainty terms are removed from the model using the proposed adaptation algorithm, Eq. (20) denoted that $s(i+1)=\rho s(i)$. Thus, if $s(i)=0, s(i+1)$ also becomes zero, and consequently $\Delta V(i+1)-\Delta V(i)=$ 0 . This means that condition (I) in Eq. (36) is feasible.

Since $1>\rho>0, s(i+1)=\rho s(i)$ implies that $s(i+1) \neq s(i)$. Thus $s(i+1) \neq s(i)$ unless $s(i+1)=s(i)=0$. This shows that the condition $(I I)$ in Eq. (36) is not feasible and only condition $(I)$ is valid. Condition $(I)$ denotes that if $s(i)=0$, not only $\Delta V(i)=0$, but also $\Delta V(i+1)-\Delta V(i)=0$. The third Lyapunov difference function is calculated as follows:

$$
\begin{gathered}
{[\Delta V(i+2)-\Delta V(i+1)]-[\Delta V(i+1)-\Delta V(i)] \approx} \\
\frac{1}{2}\left(\rho^{2}-1\right)\left(s^{2}(i+2)-2 s^{2}(i+1)+s^{2}(i)\right)
\end{gathered}
$$

Assuming a small sampling time, it can be concluded that, since $s(i)=0$ leads to $\Delta V(i)=0$, according to the DSMC reaching law, the next two time step values of $s(i), s(i+1), s(i+2)$, are also zero. Subsequently, the second and third Lyapunov difference functions become zero when $s(i)=0$, in the same manner which was discussed for the second order difference function. This is not limited to the second and third Lyapunov difference equations and can be extended to all the higher order difference functions [15]. The later conclusion is the key to proof the global asymptotic stability by invoking the new Invariance Principle for nonautonomous system which was introduced in [21].

The new Invariance Principle theorem is basically an extension to LaSalle's Invariance Principle [22] to discrete systems, in which the continuity requirement of LaSalle's Invariance Principle is removed. Unlike the Lyapunov stability analysis which focuses on negative definite condition of the Lyapunov difference function, the new Invariance Principle theorem allows to conclude asymptotic stability with respect to a negative semi definite difference function of a positive definite Lyapunov equation. It was shown that $\Delta V(i)=0$ if $s(i)=0$. Moreover, when $s(i)=0$, all the future values of the sliding variable and higher order Lyapunov differences become zero. This means that all the trajectories of the system approach the set defined by $\Delta V(i) \equiv 0$. Since the only trajectory which satisfies the nonlinear system equation is $s(i)=0$, $\tilde{\alpha}(i)=0$, and $\tilde{\beta}(i)=0$, this trajectory is a limit point and also an equilibrium point [15]. 
- Theorem 1 (The new Invariance Principle [21]): For the nonlinear dynamic system in Eq. (17), it was shown that there exits a positive definite Lyapunov function ( $V$, Eq. $(22)$ ), which its difference function $(\Delta V)$ is negative semi definite. Because the Lyapunov difference function cannot be negative for any unlimited period of time, it must be identically zero at any limit point [23]. Two new domains are defined as follows:

$$
\begin{gathered}
\Omega_{0}=\left\{x \mid V(x) \leq V\left(x_{0}\right)\right\} \\
\Omega_{i}=\{x \mid \Delta V(x) \equiv 0\}
\end{gathered}
$$

where $x_{0}=x(i=0)$ is the initial condition. Because the Lyapunov difference function is negative semi definite, it can be concluded that all system states are bounded and contained within the domain $\Omega_{0}$. If the following condition holds:

- $|x(i)+T f(x(i))+T g(x(i)) u(i)|$ is bounded for any bounded $x$,

then the only limit point (equilibrium point, $s(i)=0, \tilde{\alpha}(i)=0$, and $\tilde{\beta}(i)=0$ ) of the system belongs to $\Omega_{f}=\Omega_{0} \bigcap \Omega_{i}$. The condition above is fulfilled assuming a small enough sampling time. This means that $s(i), \tilde{\alpha}(i), \tilde{\beta}(i) \rightarrow$ 0 as $i \rightarrow \infty$. Thus, the asymptotic convergence of the three variables to zero with a positive definite Lyapunov function and a negative semi-definite Lyapunov difference equation is concluded.

Overall, the proposed adaptive robust DSMC design procedure can be summarized in the following theorem:

- Theorem 2: Consider the discrete uncertain nonlinear dynamic system in Eq. (17). If the adaptive robust DSMC input ( $\left.u_{\text {adaptive }}^{\text {mod }}\right)$ is calculated as:

$$
\begin{aligned}
& u_{\text {adaptive }}^{\text {mod }}(i)= \\
& \frac{1}{g T}\left[(\rho-1) x(i)-\rho x_{d}(i)-(\hat{\beta} f+\hat{\alpha}) T+x_{d}(i+1)\right]-\mu_{u}(i) s(i)
\end{aligned}
$$

in which, $\hat{\alpha}$ and $\hat{\beta}$ are estimated by Eq. (30) and $\mu_{u}$ is determined by the algorithm in Figure 2, then:

- The trajectories of the system will be driven to the boundary layer around the sliding manifold $(s=0)$ in finite time which gives guaranteed minimum tracking error under ADC and model uncertainties,

- The asymptotic convergence conditions of the tracking error $(s)$, and unknown parameters estimation errors $(\tilde{\alpha}, \tilde{\beta})$ to zero will be satisfied. 


\section{Case Study: Automotive Engine Cold Start Control}

The effectiveness of the adaptive control strategies for powertrain controls has been demonstrated in the literature [24, 25]. Here, a physics-based spark ignition (SI) engine model during the cold start period from [26] is used to investigate the proposed adaptive DSMC performance. The model is parameterized for a 2.4-liter, 4-cylinder, DOHC 16 valve Toyota 2AZ-FE engine. The engine rated power is $117 \mathrm{~kW} @ 5600 \mathrm{RPM}$ and it has a rated torque of $220 \mathrm{Nm} @ 4000$ RPM. The experimental validation of different components of the engine model is found in $[27,28,29]$. The model inputs are: (i) $\dot{m}_{a i}$, air mass flow rate into the intake manifold, (ii) $\dot{m}_{f c}$, commanded fuel mass flow rate, and (iii) $\Delta$, spark timing after top dead center (ATDC). The discretized model includes four difference equations:

$$
\begin{array}{r}
T_{e x h}(i+1)=\left(1-\frac{T}{\tau_{e}}\right) T_{e x h}(i)+\frac{T}{\tau_{e}}(7.5 \Delta(i)+600) A F I(i) \\
\dot{m}_{f}(i+1)=\dot{m}_{f}(i)+\frac{T}{\tau_{f}}\left[\dot{m}_{f c}(i)-\dot{m}_{f}(i)\right] \\
\omega_{e}(i+1)=\omega_{e}(i)+\frac{T}{J} T_{E}(i) \\
m_{a}(i+1)=m_{a}(i)+\left[\dot{m}_{a i}(i)-\dot{m}_{a o}(i)\right] T
\end{array}
$$

The four states are the exhaust gas temperature $\left(T_{\text {exh }}\right)$, fuel mass flow rate into the cylinders $\left(\dot{m}_{f}\right)$, the engine speed $\left(\omega_{e}\right)$, and the mass of air inside the intake manifold $\left(m_{a}\right)$. Details of the functions and constants in the engine model are available in the Appendix. The goal is to keep exhaust gas temperature, engine speed, and air-to-fuel ratio (AFR) at their desired values. Accurate and smooth tracking of the desired AFR, $T_{e x h}$, and engine speed trajectories is a key for achieving optimal engine operation, better fuel economy, and lower engine-out emissions [30, 31]. To this end, a set of four SISO DSMCs are designed.

The first sliding surface is defined for the $T_{e x h}$ controller and it is considered to be the error in tracking the desired exhaust gas temperature, which is controlled by spark timing. For the fuel flow rate controller, the desired fuel mass flow rate $\left(\dot{m}_{f, d}\right)$ is calculated from the desired AFR trajectory $\left(A F R_{d}=\frac{\dot{m}_{a o, d}}{\dot{m}_{f, d}}\right)$. Since AFR is a function of both air and fuel mass flow rates and air mass flow rate controls the engine speed, the desired fuel mass flow rate is considered as the second sliding surface. For the engine speed 
controller, the third sliding variable is defined to be the error in tracking the desired engine speed trajectory. Since there is no explicit control input for tracking the desired engine speed, desired air mass is considered as the synthetic control input for the engine speed regulation. Introducing the desired air mass requires another DSMC to track the desired air mass which means four sliding surfaces are required:

$$
\begin{gathered}
s_{1}=T_{e x h}-T_{e x h, d}, \quad s_{2}=\dot{m}_{f}-\dot{m}_{f, d} \\
s_{3}=\omega_{e}-\omega_{e, d}, \quad s_{4}=m_{a}-m_{a, d}
\end{gathered}
$$

The engine control system shown in Figure 4 is used to implement the adaptive DSMCs. As can be observed from Figure 4, $T_{e x h}, A F R\left(\dot{m}_{f}\right), m_{a}$, and $\omega_{e}$ can be measured on an engine; thus the ADC affects these four measured states and corresponding sliding surfaces. For analysis purposes, first a baseline adaptive SISO DSMC (Eq. (19) and (30)) is designed to drive the states of the system towards the desired values. Next, the predicted ADC uncertainties are incorporated into the adaptive DSMC formulation (Eq. (39)).

- Exhaust Gas Temperature Controller: According to Eq. (40), the function $f_{T_{e x h}}$ to capture dynamics of the exhaust gas temperature with additive $\left(\alpha_{T_{e x h}}\right)$ and multiplicative $\left(\beta_{T_{e x h}}\right)$ uncertainty terms is:

$$
f_{T_{e x h}}=\beta_{T_{e x h}} \times \frac{1}{\tau_{e}}\left(600 A F I-T_{e x h}\right)+\alpha_{T_{e x h}}
$$

Although in the empirical model of the exhaust gas temperature $\alpha_{T_{e x h}}$ has no physical meaning, it can present the error in the empirical model. The exhaust temperature dynamics depends heavily on the exhaust gas time constant $\left(\tau_{e}\right)$. Thus, any error in estimating the time constant $\left(\tau_{e}\right)$ results in significant deviation from the nominal model. To this end, the multiplicative uncertainty term $\left(\beta_{T_{e x h}}\right)$ is assumed to compensate for any error in estimating $\tau_{e}$. The errors in the modeled exhaust gas temperature dynamics are compensated according to the following adaptation laws with respect to Eq. (30):

$$
\begin{array}{r}
\hat{\beta}_{T_{e x h}}(i+1)=\hat{\beta}_{T_{e x h}}(i)+\frac{T\left(s_{1}(i)\right)}{\tau_{e} \rho_{\beta_{1}}}\left(600 A F I-T_{e x h}(i)\right) \\
\hat{\alpha}_{T_{e x h}}(i+1)=\hat{\alpha}_{T_{e x h}}(i)+\frac{T\left(T_{e x h}(i)-T_{e x h, d}(i)\right)}{\rho_{\alpha_{1}}}
\end{array}
$$




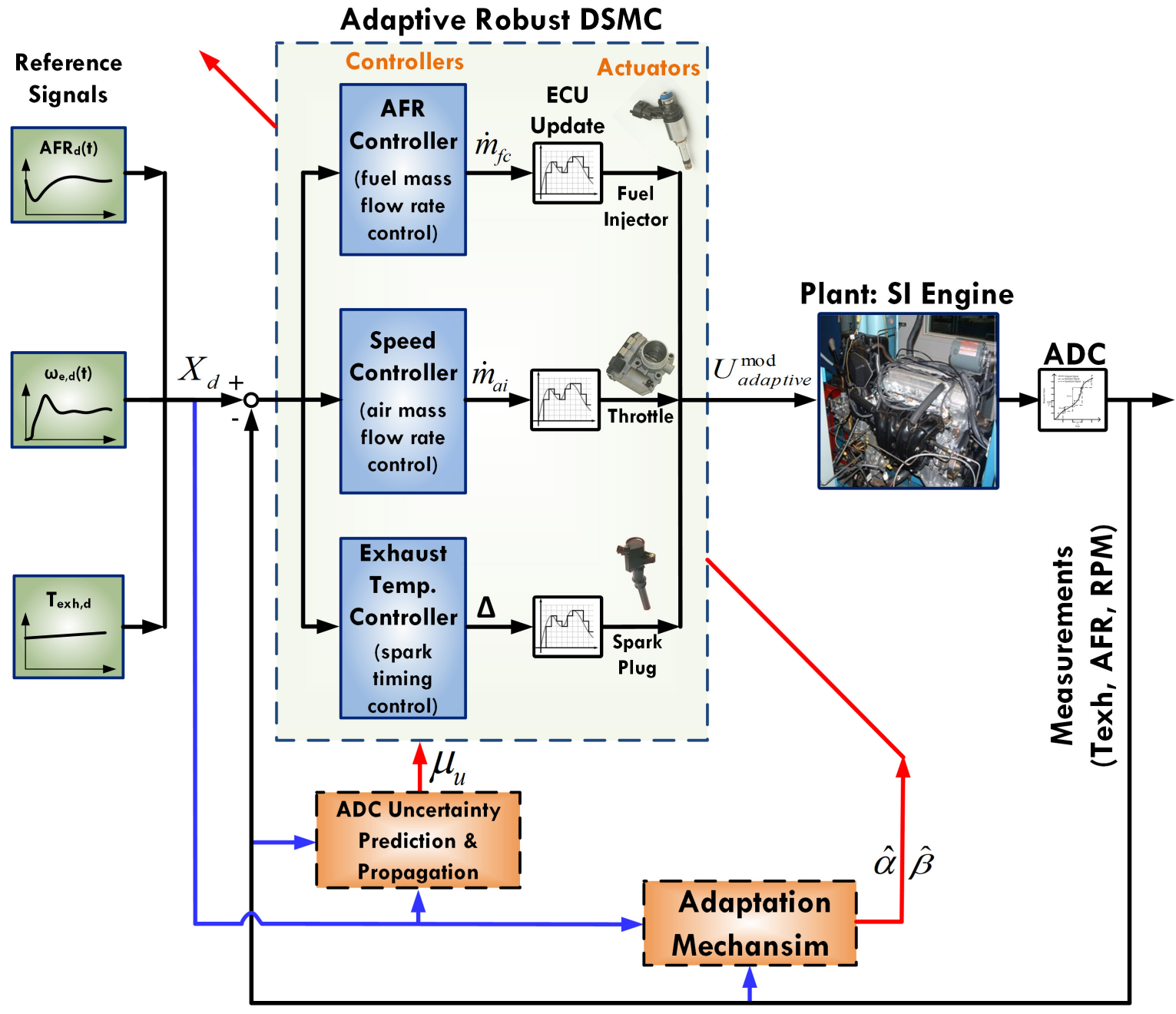

Figure 4: Block diagram of the engine cold start control system.

By incorporating the solutions of Eq. (43) and the predicted implementation imprecisions $\left(\mu_{\Delta}\right)$ into Eq. (39), the modified adaptive DSMC for exhaust temperature becomes:

$$
\begin{aligned}
\Delta(i)= & \frac{\tau_{e}}{7.5 A F I . T}\left[-\hat{\beta}_{T_{e x h}} \frac{T}{\tau_{e}}\left(600 A F I-T_{e x h}(i)\right)+\hat{\alpha}_{T_{e x h}}(i)\right. \\
& \left.+\left(\rho_{1}-1\right) s_{1}(i)+T_{e x h, d}(i+1)-T_{e x h, d}(i)\right]-\mu_{\Delta}(i) s_{1}(i)
\end{aligned}
$$

if $\mu_{\Delta}=0$ the controller is an adaptive baseline DSMC, while when $\mu_{\Delta}$ is calculated according to the mechanism in Figure 2, the controller is an 
integrated adaptive DSMC with predicted ADC uncertainties.

- Fuel Flow Rate Controller: As can be observed from Eq. (40), the fuel flow dynamics $\left(f_{\dot{m}_{f}}\right)$ with additive $\left(\alpha_{\dot{m}_{f}}\right)$ and multiplicative $\left(\beta_{\dot{m}_{f}}\right)$ uncertainty terms becomes:

$$
f_{\dot{m}_{f}}=-\beta_{\dot{m}_{f}} \times \frac{1}{\tau_{f}}\left(\dot{m}_{f}(i)\right)+\alpha_{\dot{m}_{f}}
$$

In practice, $\dot{m}_{f}$ is not measured directly and it is calculated according to the AFR sensor measurement and estimated air mass inside the cylinder $\left(\dot{m}_{a o}\right) . \quad \alpha_{\dot{m}_{f}}$ represents the error in estimating fuel flow rate due to AFR measurement uncertainty and error in predicting the air mass. Similar to exhaust gas temperature dynamics, the fuel evaporation time constant $\tau_{f}$ dictates the dynamics of the fuel flow into the cylinder. Thus, any error in estimating the time constant $\left(\tau_{f}\right)$ results in significant deviation from the nominal model. To this end, the multiplicative uncertainty is introduced to the fuel flow dynamics to represent the uncertainty in estimating $\tau_{f}$. The adaptation laws for $\beta_{\dot{m}_{f}}$ and $\alpha_{\dot{m}_{f}}$ are:

$$
\begin{array}{r}
\hat{\beta}_{\dot{m}_{f}}(i+1)=\hat{\beta}_{\dot{m}_{f}}(i)-\frac{T\left(s_{2}(i)\right)}{\tau_{f} \rho_{\beta_{2}}} \dot{m}_{f}(i) \\
\hat{\alpha}_{\dot{m}_{f}}(i+1)=\hat{\alpha}_{\dot{m}_{f}}(i)+\frac{T\left(\dot{m}_{f}(i)-\dot{m}_{f, d}(i)\right)}{\rho_{\alpha_{2}}}
\end{array}
$$

where $\dot{m}_{f, d}$ is calculated according to the desired AFR. The adaptive control law for fuel flow rate into the cylinder with multiplicative and additive unknown terms and the predicted ADC imprecisions $\left(\mu_{\dot{m}_{f c}}\right)$ is:

$$
\begin{array}{r}
\dot{m}_{f c}(i)=\frac{\tau_{f}}{T}\left[\hat{\beta}_{\dot{m}_{f}} \frac{T}{\tau_{f}}\left(\dot{m}_{f}(i)\right)+\hat{\alpha}_{\dot{m}_{f}}(i)+\left(\rho_{2}-1\right) s_{2}(i)\right. \\
\left.+\dot{m}_{f, d}(i+1)-\dot{m}_{f, d}(i)\right]-\mu_{\dot{m}_{f c}}(i) s_{2}(i)
\end{array}
$$

- Engine Speed Controller: $f_{\omega_{e}}$ for the engine with multiplicative $\left(\beta_{\omega_{e}}\right)$ and additive $\left(\alpha_{\omega_{e}}\right)$ unknown parameters is:

$$
f_{\omega_{e}}=-\beta_{\omega_{e}} \times \frac{1}{J}\left(T_{\text {loss }}\right)+\alpha_{\omega_{e}}
$$

where $T_{\text {loss }}=0.4 \omega_{e}+100 . T_{\text {loss }}$ represents the torque losses (e.g., due to friction) on the crankshaft. Thus, the additive uncertainty $\alpha_{\omega_{e}}$ represents the 
error in reading the torque map. Since reading the values from the torque map between break points in the look-up table uses a linear relationship, the additive uncertainty term can express the potential error in the modeled dynamics accordingly. Also $\beta_{\omega_{e}}$ represent the uncertainty in estimating the effective engine inertia $(J)$. $\beta_{\omega_{e}}$ and $\alpha_{\omega_{e}}$ are driven to zero using the following adaptation laws:

$$
\begin{array}{r}
\hat{\beta}_{\omega_{e}}(i+1)=\hat{\beta}_{\omega_{e}}(i)-\frac{T\left(s_{3}(i)\right)}{J \rho_{\beta_{3}}}\left(0.4 \omega_{e}(i)+100\right) \\
\hat{\alpha}_{\omega_{e}}(i+1)=\hat{\alpha}_{\omega_{e}}(i)+\frac{T\left(\omega_{e}(i)-\omega_{e, d}(i)\right)}{\rho_{\alpha_{3}}}
\end{array}
$$

Finally, the control input $\left(m_{a, d}\right)$ for engine speed regulation after incorporating the propagated ADC uncertainties $\left(\mu_{m_{a, d}}\right)$ yields:

$$
\begin{gathered}
m_{a, d}(i)=\frac{J}{30,000 T}\left[\hat{\beta}_{\omega_{e}} \frac{T}{J}\left(100+0.4 \omega_{e}(i)\right)+\hat{\alpha}_{\omega_{e}}(i)\right. \\
\left.+\left(\rho_{3}-1\right) s_{3}(i)+\omega_{e, d}(i+1)-\omega_{e, d}(i)\right]-\mu_{m_{a, d}}(i) s_{3}(i)
\end{gathered}
$$

- Air Mass Flow Controller: The intake air manifold dynamics are linked to the rotational dynamics through the calculated $m_{a, d}$. The calculated $m_{a, d}$ from Eq. (50) is used as the desired trajectory to obtain $\dot{m}_{a i}$ as the control input of the intake air flow rate controller. The intake manifold air mass dynamics with additive model uncertainty $\left(\alpha_{m_{a}}\right)$ and the multiplicative unknown parameter $\left(\beta_{m_{a}}\right)$ is:

$$
f_{m_{a}}=-\beta_{m_{a}}\left(\dot{m}_{a o}(i)\right)+\alpha_{m_{a}}
$$

where [26]:

$$
\begin{gathered}
\dot{m}_{a o}=k_{1} \eta_{v o l} m_{a} \omega_{e} \\
\eta_{v o l}=m_{a}^{2}\left(k_{2} \omega_{e}^{2}+\right. \\
\left.k_{3} \omega_{e}+k_{4}\right)+m_{a}\left(k_{5} \omega_{e}^{2}+k_{6} \omega_{e}+k_{7}\right) \\
+k_{8} \omega_{e}^{2}+k_{9} \omega_{e}+k_{10}
\end{gathered}
$$

$k_{1,2, \ldots, 10}$ are the empirical parameters of the volumetric efficiency $\left(\eta_{\text {vol }}\right)$ curve fit. As shown in Eq. (51)-(53), the uncertainty terms in the intake air manifold dynamics $\left(\alpha_{m_{a}}\right.$ and $\left.\beta_{m_{a}}\right)$ compensate for the uncertainties in $\dot{m}_{a o}$ which is read through the $\eta_{v o l}$ curve fit. $\beta_{m_{a}}$ and $\alpha_{m_{a}}$ are updated using the following 
adaptation laws:

$$
\begin{array}{r}
\hat{\beta}_{m_{a}}(i+1)=\hat{\beta}_{m_{a}}(i)-\frac{T\left(s_{4}(i)\right)}{\rho_{\beta_{4}}} \dot{m}_{a o} \\
\hat{\alpha}_{m_{a}}(i+1)=\hat{\alpha}_{m_{a}}(i)+\frac{T\left(m_{a}(i)-m_{a, d}(i)\right)}{\rho_{\alpha_{4}}}
\end{array}
$$

After incorporating the predicted ADC imprecisions $\left(\mu_{\dot{m}_{a i}}\right)$ into the air mass controller, the adaptive robust controller input is:

$$
\begin{aligned}
& \dot{m}_{a i}(i)=\frac{1}{T}\left[T \hat{\beta}_{m_{a}}\left(\dot{m}_{a o}(i)\right)+\hat{\alpha}_{m_{a}}(i)+\left(\rho_{4}-1\right) s_{4}(i)\right. \\
& \left.\quad+m_{a, d}(i+1)-m_{a, d}(i)\right]-\mu_{\dot{m}_{a i}}(i) s_{4}(i)
\end{aligned}
$$

In Eq. (44), (47), (50), and (55), $\mu_{\Delta}, \mu_{\dot{m}_{f c}}, \mu_{m_{a, d}}$, and $\mu_{\dot{m}_{a i}}$ are the estimations of propagated ADC uncertainties on control signals which are computed according to the approach explained in Figure 2. Estimation of propagated $\mathrm{ADC}$ uncertainties on control signals requires the knowledge of ADC uncertainty on measured signals $\left(\mu_{T_{e x h}}, \mu_{\dot{m}_{f}}, \mu_{\omega_{e}}\right.$, and $\left.\mu_{m_{a}}\right)$ that are predicted using Eq. (11). The verification results of the estimated ADC uncertainties of the engine plant are summarized in Figure 5 and Table 1 for a set of measured $T_{e x h}, \dot{m}_{f}, \omega_{e}$, and $m_{a}$ signals under $10 \mathrm{~ms}$ of sampling time and quantization level of 10-bit. For better evaluation of the uncertainty prediction mechanism, the measured engine signals are defined in their worst and nonsmooth shapes. The high accuracy of the ADC uncertainty prediction mechanism can be observed for the engine case study from Figure 5 and Table 1.

Table 1: Mean $(\bar{e})$ and standard deviation $\left(\sigma_{e}\right)$ of ADC uncertainty prediction errors for the SI engine model.

\begin{tabular}{lcc}
\hline \hline & $\bar{e}$ & $\sigma_{e}$ \\
\hline$T_{\text {exh }}\left[{ }^{\circ} \mathrm{C}\right]$ & 0.5 & 0.5 \\
$\dot{m}_{f}[\mathrm{~kg} / \mathrm{sec}]$ & $2.337 \times 10^{-6}$ & $2.732 \times 10^{-6}$ \\
$\omega_{e}[\mathrm{rad} / \mathrm{sec}]$ & 0.124 & 0.145 \\
$m_{a}[\mathrm{~kg}]$ & $2.060 \times 10^{-5}$ & $2.321 \times 10^{-5}$ \\
\hline \hline
\end{tabular}

The impact of unknown additive and multiplicative terms on part of the baseline engine DSMC that is associated with the plant's dynamics $(f)$ is 


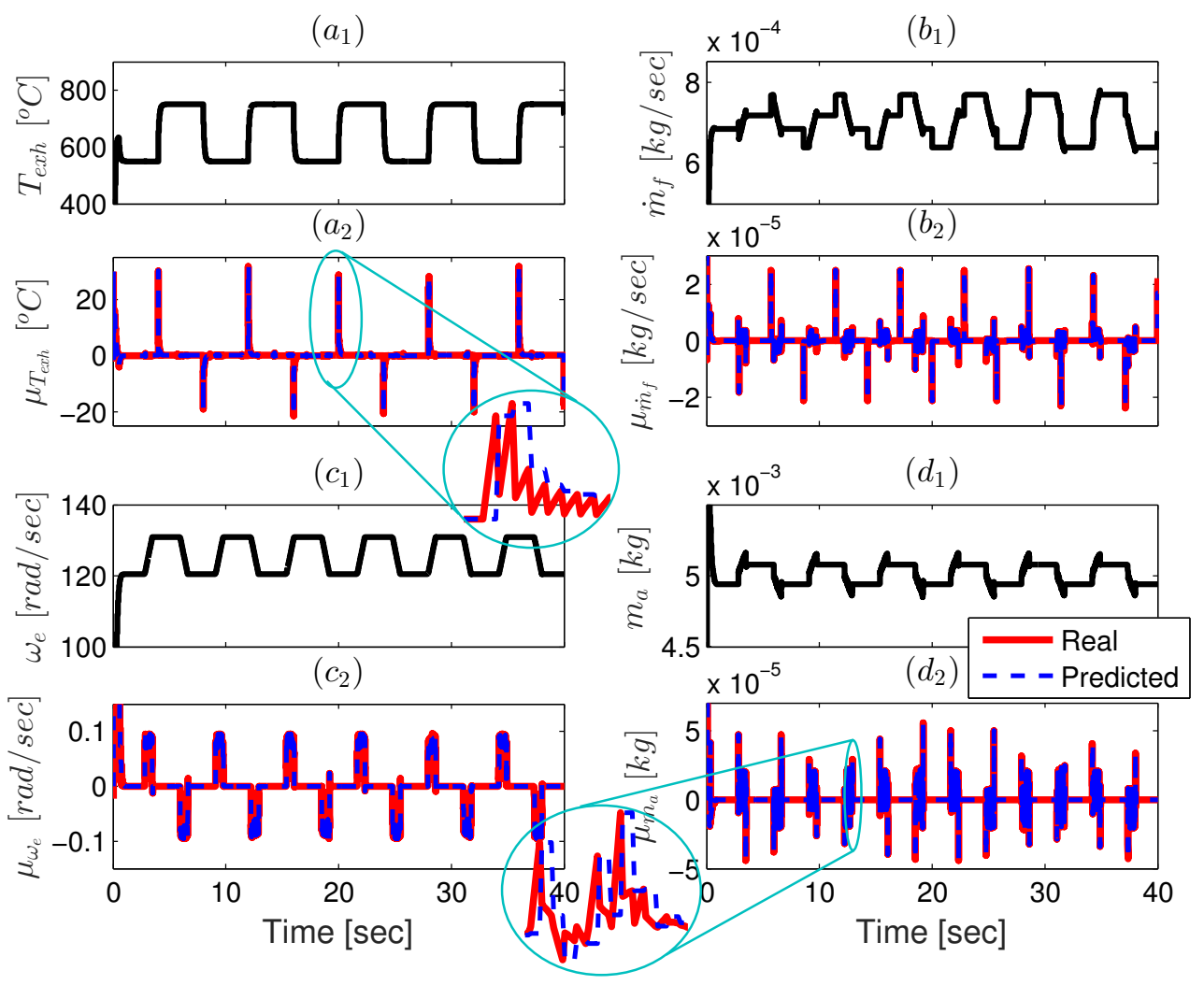

Figure 5: Comparison of actual and predicted uncertainties due to sampling and quantization on measured variables of the SI engine: (a) $T_{e x h}$ (b) $\dot{m}_{f}$, (c) $\omega_{e}$, and (d) $m_{a}$ (10 $m s$ sampling time and 10-bit quantization level).

shown in Figure 6. The multiplicative terms $(\beta)$ for the engine case study were assumed to be \pm 0.75 , which means up to $25 \%$ error on each of the states dynamics. The equivalent additive unknown terms $(\alpha)$, for which the dynamics show a similar deviation from the nominal model due to corresponding multiplicative unknown terms, were found for every single dynamic. In the absence of the adaptation, the selected values for the additive unknown terms result in the same errors in the dynamics which were already observed from the multiplicative terms.

The uncertainties on the model parameters cause a permanent error in the estimated dynamics compared to the nominal model (model with no uncertainty). These errors significantly demolish the DSMC tracking performance and consequently, the outcome of the non-adaptive DSMC will not be accept- 
(a)
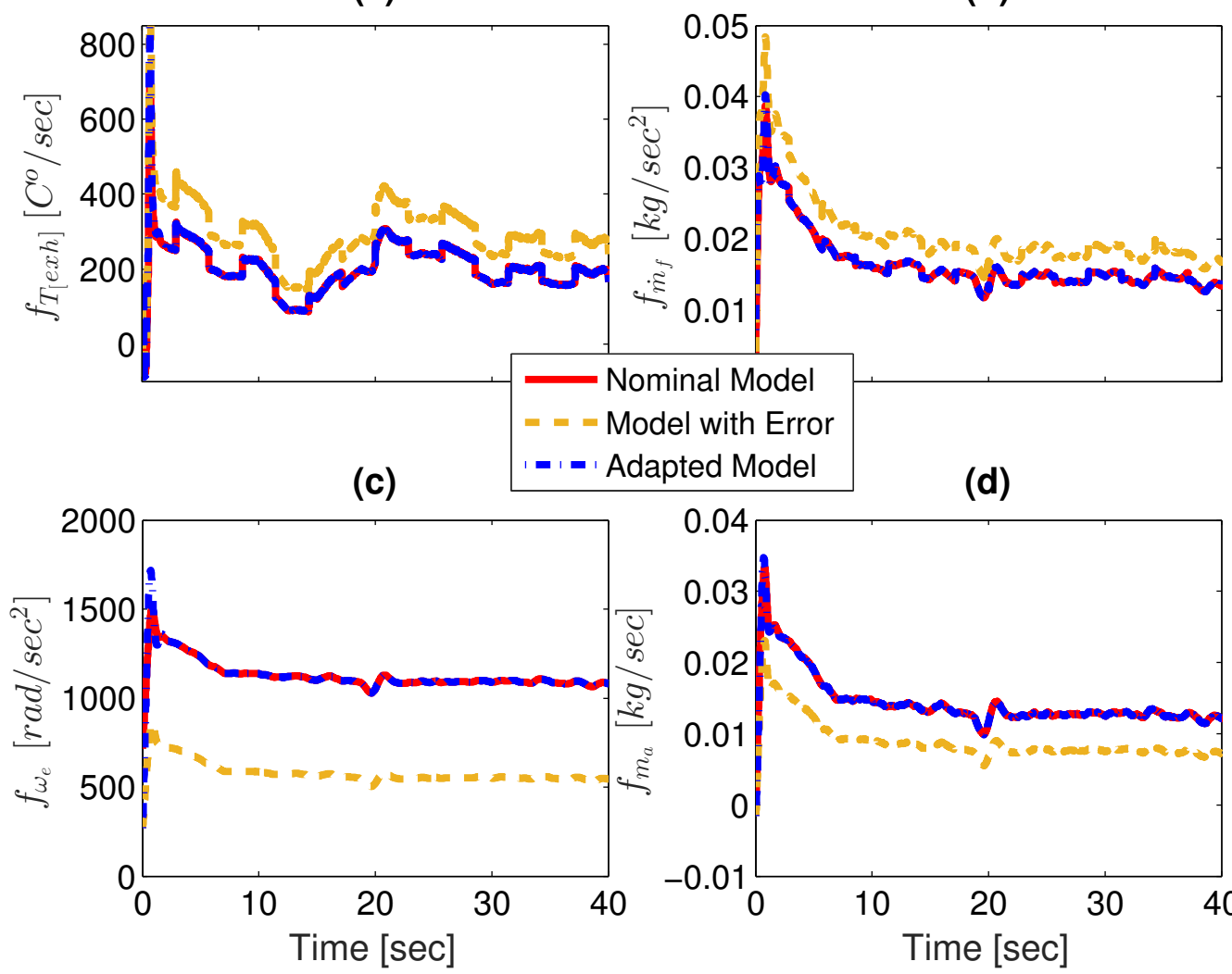

(b)

(d)

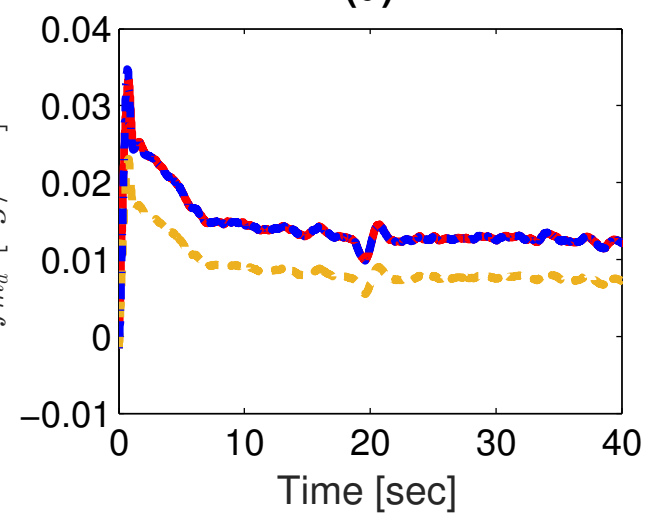

Figure 6: The impacts of unknown uncertainty terms on the engine dynamics inside the DSMC and how the adaptation mechanisms drive the model with error to its nominal value: (a) $T_{e x h}$, (b) $\dot{m}_{f}$, (c) $\omega_{e}$, and (d) $m_{a}$ (10 ms sampling time and 10-bit quantization level).

able anymore. Upon activation of the adaptation mechanisms (Eq. (30)), it can be seen from Figure 6 that the model with error converges to the nominal model and the model uncertainties are compensated. Figure 7 shows the results of unknown multiplicative and additive uncertainty terms estimation against the actual (nominal) values. Despite the short time period (40 sec) during which the engine cold start controller should operate, the adaptation mechanism removes the errors in the modeled dynamics in the DSMC in less than $2 \mathrm{sec}$ by driving the unknown multiplicative and additive parameters to their nominal values, "1" and "0", respectively.

Figure 8 shows the impact of errors in the engine's model on the DSMC tracking performance. As it is expected, the non-adaptive DMSC fails to 
$\left(a_{1}\right)$

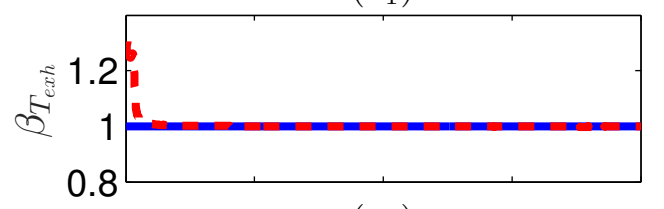

$\left(a_{2}\right)$
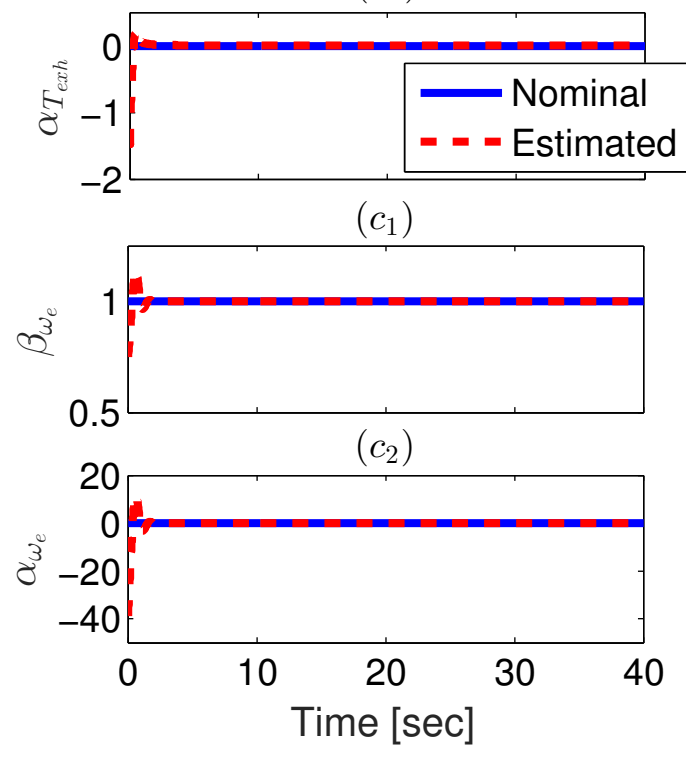

$\left(b_{1}\right)$
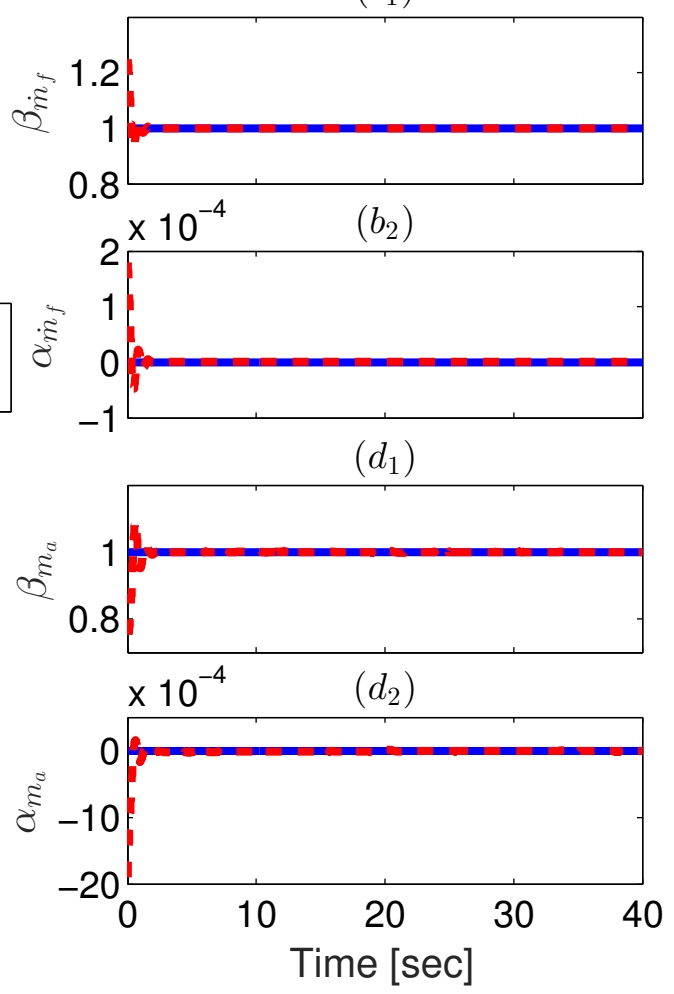

Figure 7: Convergence results of unknown engine model multiplicative and additive terms (10 ms sampling time and 10-bit quantization level).

track the desired trajectories, which explains the importance of handling the model uncertainties in the body of the proposed DSMC. On the other hand, once the adaptation algorithms are activated and the convergence period of the unknown parameters is over, the baseline adaptive DSMC tracks all the desired trajectories smoothly with the minimum error under $10 \mathrm{~ms}$ of sampling time and quantization level of 10-bit. Table 2 summarizes the effect of unknown parameter variations (25\% error from each type of model uncertainties) on the tracking error (Table 2-a), and dynamic responses (Table 2-b) for baseline non-adaptive and adaptive DSMCs. By comparing the errors in trajectory tracking and dynamic estimation of the non-adaptive DSMC with those from the adaptive DSMC, one can conclude the effectiveness of the proposed adaptation mechanism in handling the model uncertainties for all cases. It can be observed that the adaptive DSMC enhances the trajectory tracking errors by more than $95 \%$ compared to the non-adaptive DSMC. 
(a)

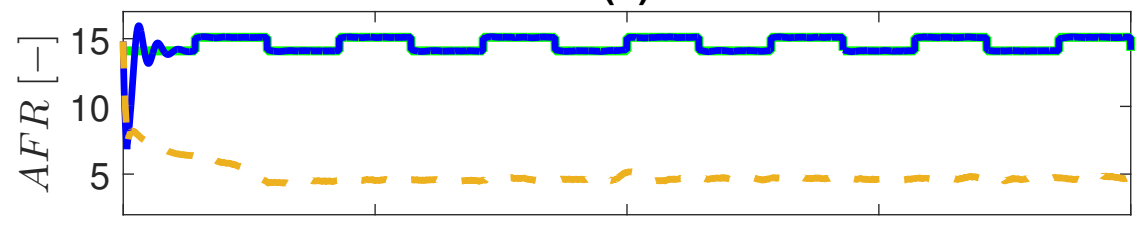

(b)
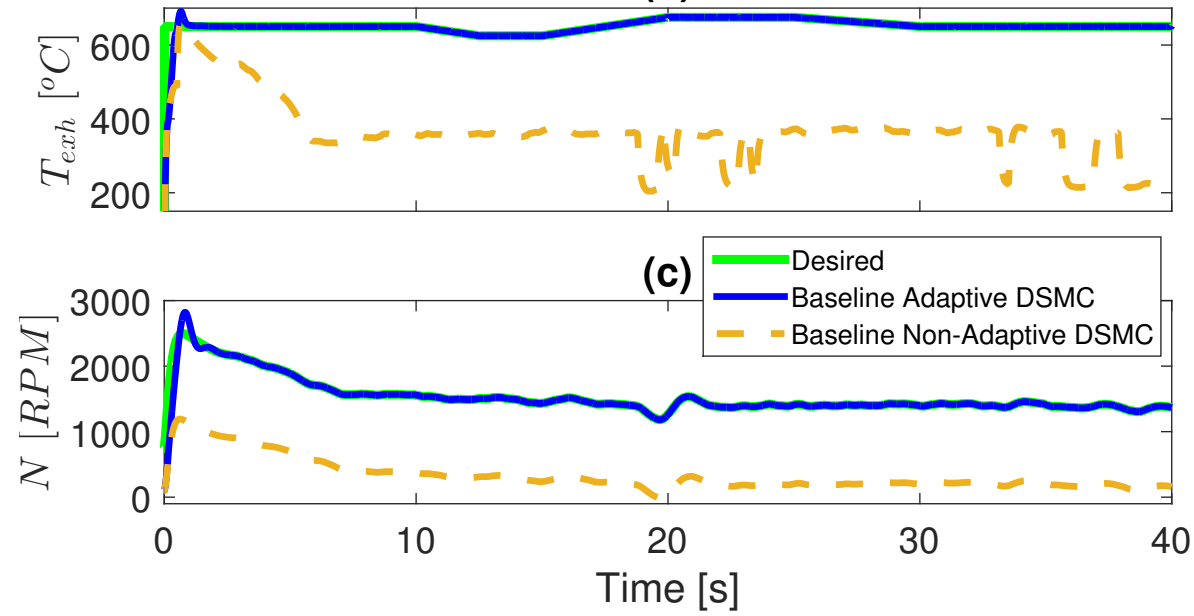

Figure 8: Comparison between baseline adaptive and non-adaptive engine DSMCs under ADC imprecisions and model uncertainties: (a) air-fuel ratio, (b) exhaust gas temperature, and (c) engine speed (10 $\mathrm{ms}$ sampling time and 10-bit quantization level).

The better tracking performance of the adaptive controller is a direct result of the accurate and fast estimation of the unknown parameters by the adaptation technique. The adaptation mechanism removes the errors in the engine dynamics by up to $98 \%$.

In the next stage, the predicted implementation imprecisions are incorporated in the adaptive DSMC to compare the performance of the baseline adaptive controller versus the adaptive DSMC with predicted ADC uncertainties. Figure 9 shows the overall DSMC performance in tracking the desired $A F R$, exhaust gas temperature, and engine speed trajectories in the presence of unknown uncertainty terms and under $10 \mathrm{~ms}$ of sampling time and quantization level of 10-bit. It can be observed from Figure 9 that after the adaptation mechanisms removed the model uncertainties within $t=2 \mathrm{sec}$, the DMSC tracks all the desired trajectories desirably. The required time for the adaptation laws to remove the errors in the model is affected by the sampling time. It was observed that convergence of the unknown parameters 
Table 2: Mean $(\bar{e})$ value of the errors in (a) trajectory tracking (Figure 8), and (b) estimating the engine dynamics (Figure 6), from the non-adaptive and adaptive DSMCs under $25 \%$ uncertainty in each of the multiplicative $(\beta)$ and additive $(\alpha)$ unknown terms in the engine dynamics (Values inside the parentheses show the resulting improvement from the robust DSMC compared to The baseline DSMC, $T=10 \mathrm{~ms}$, quantization level=10-bit).

(a)

(b)

\begin{tabular}{|c|c|c|c|c|c|}
\hline & \multicolumn{2}{|c|}{$\overline{\bar{e}}$ (Tracking Error) } & & \multicolumn{2}{|c|}{$\bar{e}($ Model Error $)$} \\
\hline & \multirow{3}{*}{$\begin{array}{c}\text { Non-adaptive } \\
\text { DSMC } \\
\text { Reference }\end{array}$} & \multirow{3}{*}{$\begin{array}{l}\text { Adaptive } \\
\text { DSMC }\end{array}$} & & Non-adaptive & Adaptive \\
\hline & & & & DSMC & DSMC \\
\hline & & & & 0.0042 & 0.000086 \\
\hline \multirow{2}{*}{$\begin{array}{l}\text { AFR } \\
{[-]}\end{array}$} & \multirow[t]{2}{*}{9.566} & \multirow{2}{*}{$\begin{array}{c}0.103 \\
(-98.92 \%)\end{array}$} & {$\left[\mathrm{kg} / \mathrm{sec}^{2}\right]$} & & $(-97.95 \%)$ \\
\hline & & & $f_{T_{e x h}}$ & 87.1 & 1.8 \\
\hline \multirow{2}{*}{$\begin{array}{l}T_{e x h} \\
{\left[{ }^{o} C\right]}\end{array}$} & \multirow[t]{2}{*}{292.2} & \multirow{2}{*}{$\begin{array}{c}3.6 \\
(-98.8 \%) \\
\end{array}$} & {$\left[{ }^{\circ} \mathrm{C} / \mathrm{sec}\right]$} & & $(-97.8 \%)$ \\
\hline & & & & 547.0 & 7.6 \\
\hline \multirow{3}{*}{$\begin{array}{l}N \\
{[R P M]}\end{array}$} & \multirow[t]{3}{*}{1202.9} & \multirow{3}{*}{$\begin{array}{c}17.6 \\
(-98.5 \%)\end{array}$} & {$\left[\mathrm{rad} / \mathrm{sec}^{2}\right]$} & & $(-98.6 \%)$ \\
\hline & & & & 0.0055 & 0.000082 \\
\hline & & & & & $(-98.50 \%)$ \\
\hline
\end{tabular}

can be achieved in less than 200 time instants. Slower convergence rate of the unknown parameters may cause a lag in the controller tracking results, however incorporation of the ADC uncertainties knowledge helps to improve the robustness against sampling time and lowers the required computation time.

Figure 9 shows that ADC with sampling time of $10 \mathrm{~ms}$ and quantization level of 10-bit does not affect the baseline DSMC performance significantly. However, as can be observed from Figure 10, once the sampling time increases, or when the quantization level becomes smaller, the tracking errors from the baseline adaptive DSMC increase dramatically. On the other side, the proposed adaptive DSMC with incorporated ADC imprecisions is more robust to the sampling time increase and also the quantization level decrease.

$A F R$ is the most important trajectory due to its effects on engine performance in meeting the desired emission and fuel consumption targets [6]. It can be seen from Figure 10 that when the signals at the controller I/O are sampled at a higher rate than $10 \mathrm{~ms}$, the baseline adaptive DSMC shows weak performance in tracking the desired AFR trajectory. The desired AFR tracking error rises by more than 10 times when the sampling time increases from $10 \mathrm{~ms}$ to $35 \mathrm{~ms}$. Meanwhile, the adaptive DSMC with predicted ADC 
(a)
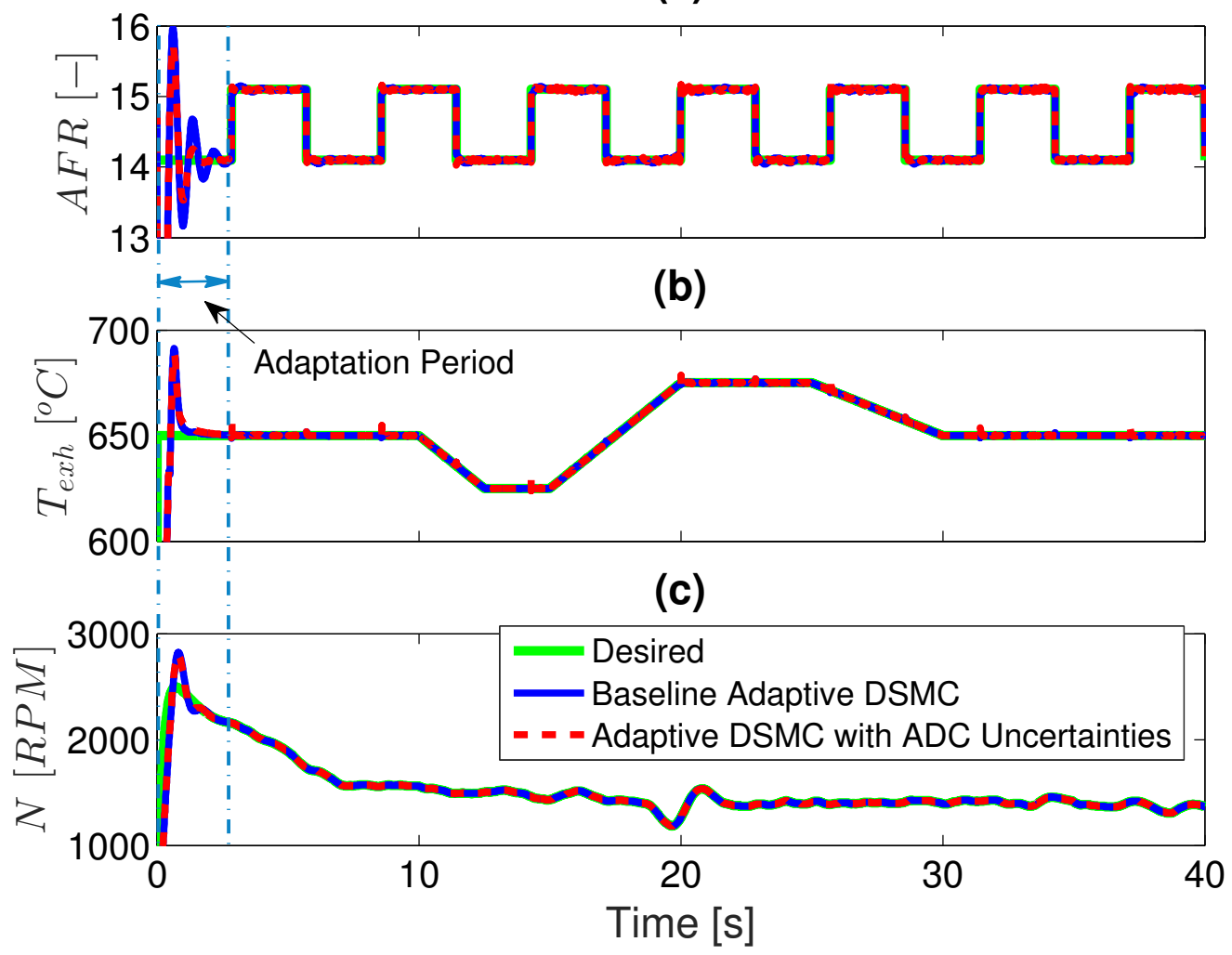

Figure 9: Results of engine control under ADC imprecisions and model uncertainties: (a) air-fuel ratio, (b) exhaust gas temperature, and (c) engine speed (10 ms sampling time and 10-bit quantization level).

uncertainties keeps the tracking error less than 0.1, despite the increase in the sampling time. This means an overall $50 \%$ decrease in AFR tracking error compared to the baseline adaptive DSMC. Similar improvement trends can be concluded from $T_{e x h}$ and $\omega_{e}$ adaptive DSMCs in which the tracking errors of the baseline controllers at higher sampling times are decreased by $50-60 \%$ upon incorporating the predicted ADC uncertainties.

Figure 11 provides an overall comparison among the baseline non-adaptive DSMC, baseline adaptive DSMC, and robust adaptive DSMC with predicted ADC uncertainties. When there is no uncertainty in the model, it can be observed that by incorporating the predicted ADC uncertainties, comparing to the baseline controller, the controller becomes more robust against sampling and quantization (sampling time of $10 \mathrm{~ms}$ and quantization level of 10-bit) imprecisions by more than $50 \%$ on average in terms of the mean tracking er- 
$\left(a_{1}\right)$

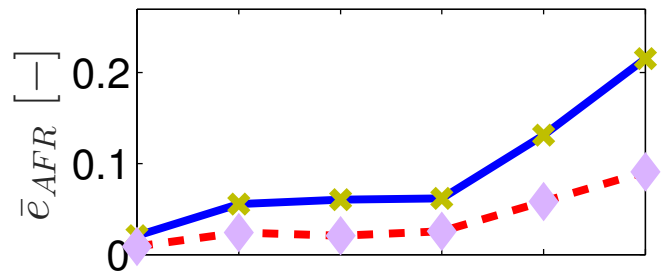

$\left(a_{2}\right)$

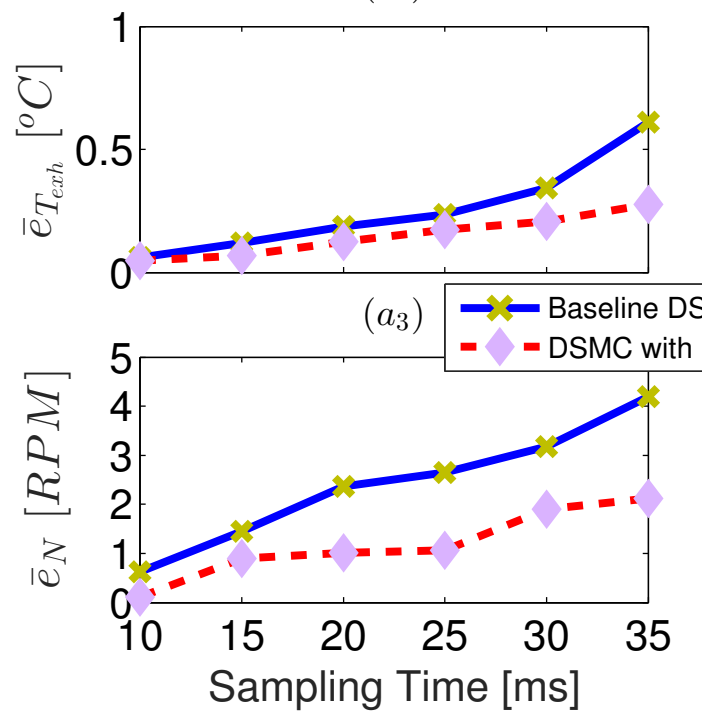

$\left(b_{1}\right)$

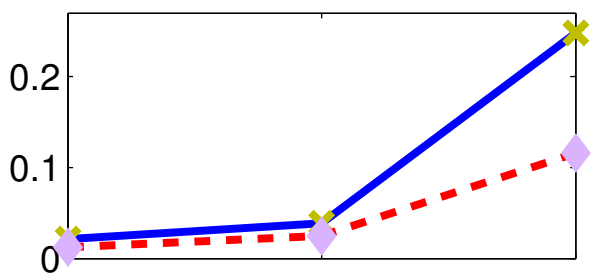

$\left(b_{2}\right)$

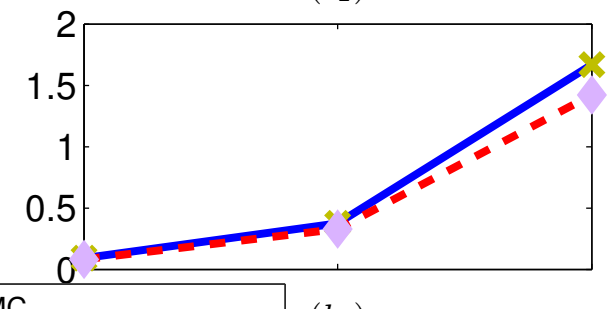

$\left(b_{3}\right)$

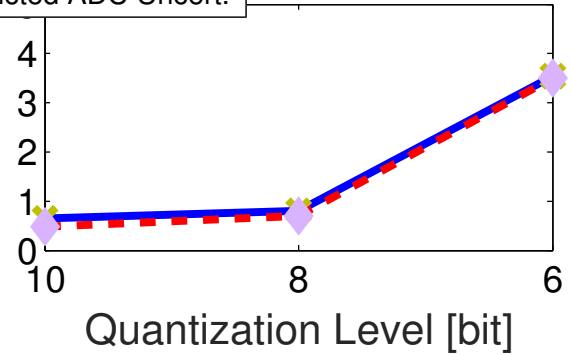

Figure 10: Effect of sampling time and quantization level on the tracking performance of the engine controller with and without the predicted ADC uncertainties.

rors (Figure 11- $a_{1}, b_{1}, c_{1}$ ). By introducing uncertainties to the engine model, both adaptive and robust adaptive DSMCs show significantly better performances, compared to the baseline DSMC (Figure 11- $a_{2}, b_{2}, c_{2}$ ). Moreover, in the presence of model and ADC uncertainties, the robust adaptive DSMC shows lower errors by 50-60\%, compared to the baseline adaptive DSMC for all cases (Figure 11- $a_{3}, b_{3}, c_{3}$ ).

The required computational time for the engine adaptive DSMC was also studied for various controller configurations using the MATLAB/Simulink ${ }^{\circledR}$ MIL platform. The MIL testing was carried out on a desktop computer with an Intel ${ }^{\circledR}$ Core $^{T M}$ i5 @ $3.20 G H z$ processor. It was observed that the average computational time of the baseline DSMC for controlling the engine during the first $40 \mathrm{sec}$ of its operation is $3.012 \mathrm{sec}$. These computational times for 


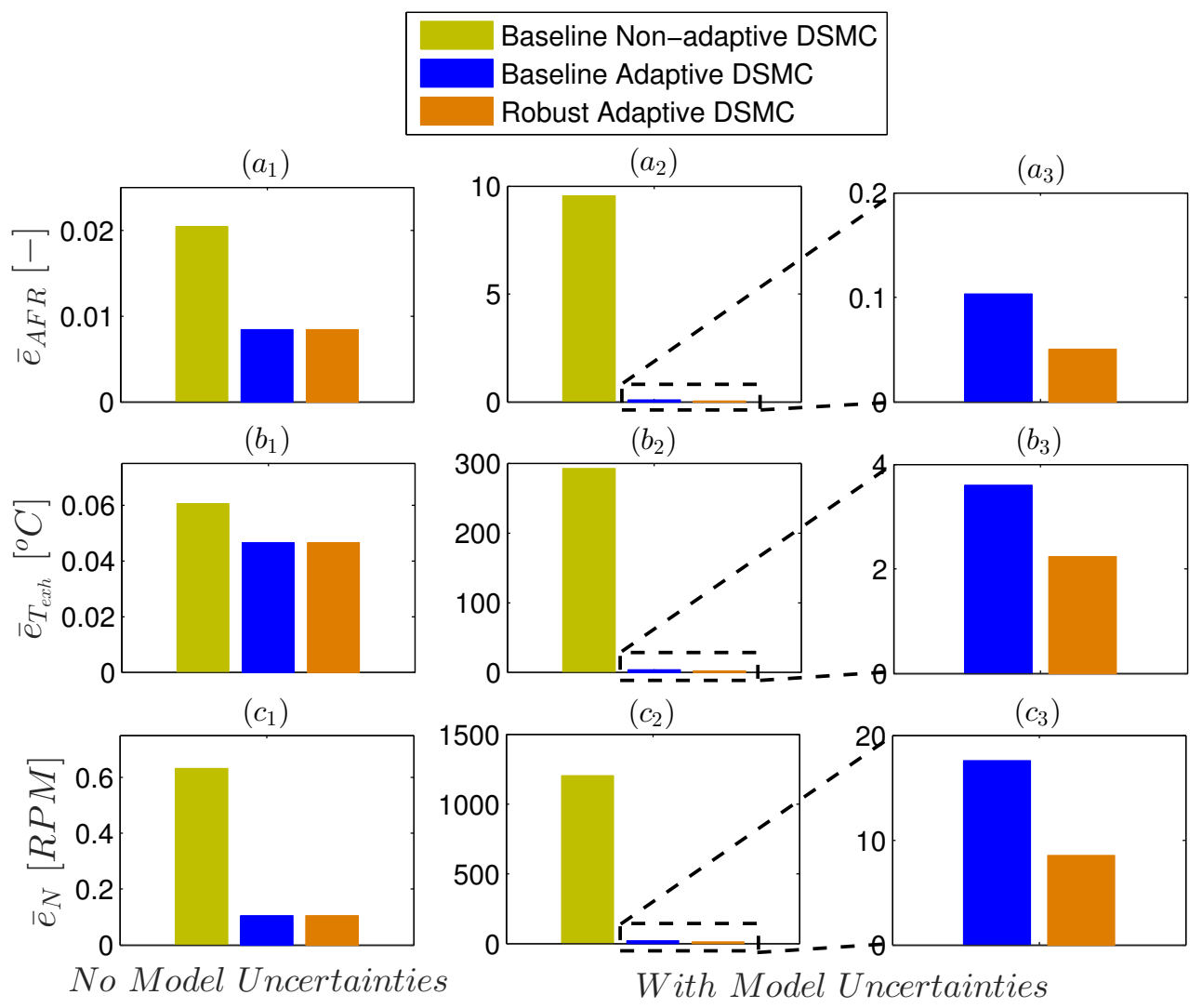

Figure 11: Effects of incorporating the predicted ADC uncertainties and the adaptation laws on the DSMC robustness characteristics under $25 \%$ uncertainty in each of the multiplicative $(\beta)$ and additive $(\alpha)$ unknown terms, and $10 \mathrm{~ms}$ sampling time and 10-bit quantization level.

the adaptive DSMCs when (i) both of the adaptation laws are active, (ii) only the multiplicative adaptation law is active, and (iii) only the additive adaptation law is active, are $3.062 \mathrm{sec}, 3.036 \mathrm{sec}$, and $3.041 \mathrm{sec}$, respectively. The computational time of the adaptive DSMCs are slightly higher than the baseline DSMC $(<2 \%)$ and this shows the effectiveness of the proposed adaptation algorithm that allows for real-time implementation.

In the engine model (Eq. (40)), the time constants in the fuel mass flow $\left(\tau_{f}\right)$ and exhaust gas temperature $\left(\tau_{e}\right)$ dynamics take into account empirical time delays within the physical engine dynamics. However, the time delay in the hardware and associated uncertainty are shown in the literature that can be addressed through SMC design [32, 33, 34]. Since most of 
the recent works in the literature are limited to linear or continuous time systems, extending the proposed adaptive robust DSMC design to nonlinear time delayed systems needs to be investigated in future studies.

\section{DSMC Real-Time Verification}

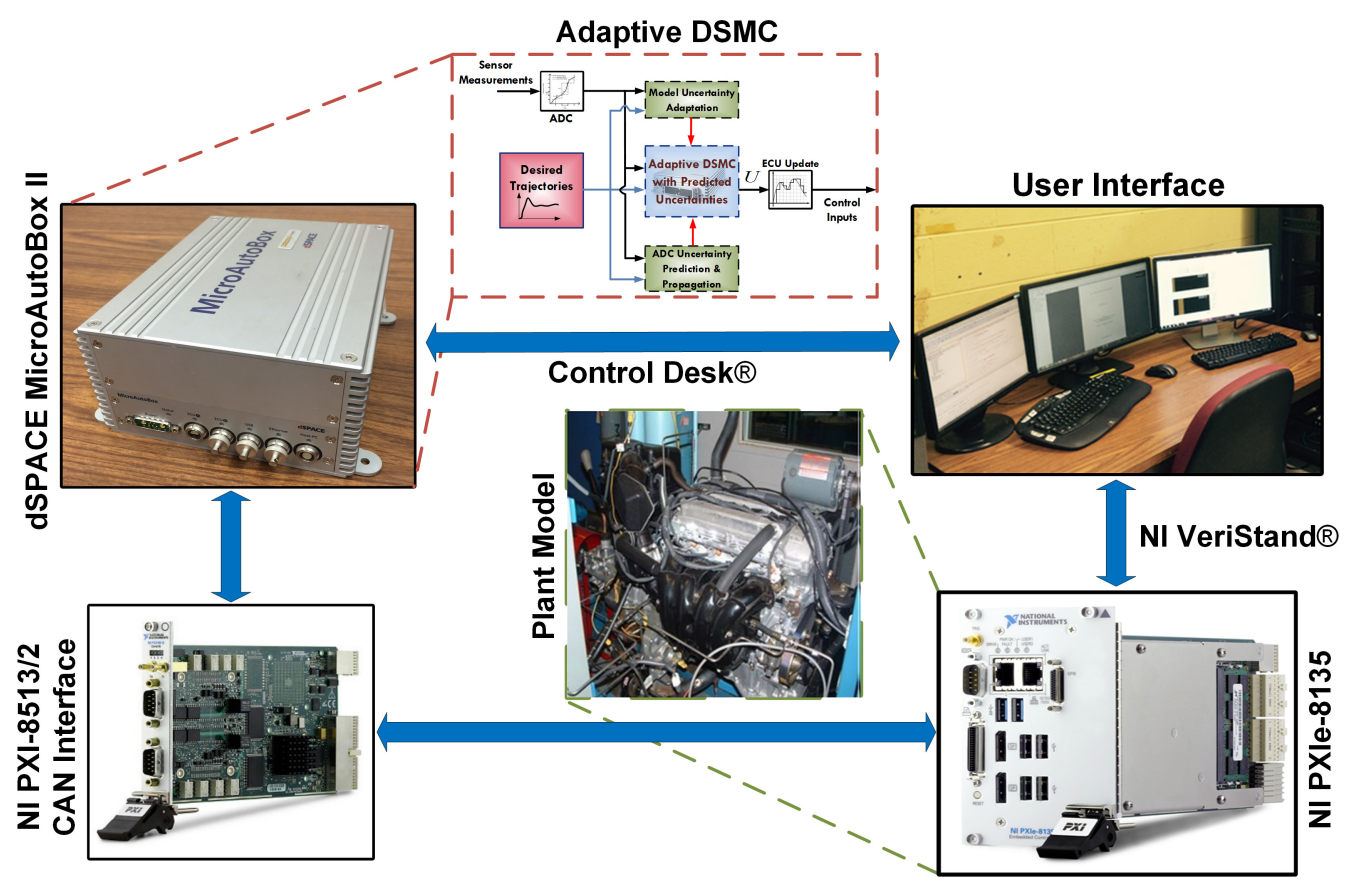

Figure 12: Schematic of the designed PIL setup for real-time DSMC verification.

The designed controllers in the previous section with adaptation mechanisms are tested in a PIL setup, shown in Figure 12, to verify the performances of the adaptive DSMC in real-time. Two processors are employed in the PIL setup. The first one is a National Instrument (NI) PXI processor (NI PXIe-8135) in which the engine plant model is implemented. The second processor is a dSPACE MicroAutobox II (MABX) which is the main ECU. The generated C-code of the adaptive DSMC along with the adaptation and uncertainty prediction mechanisms are implemented into MABX. The trajectory tracking performances and the engine operation are monitored in real-time under embedded ADC imprecisions on feedback and control signals, in the presence of additive and multiplicative types of model 
uncertainties in the controller. Real-time test configuration is conducted using NI VeriStand ${ }^{\circledR}$ and dSPACE Control Desk ${ }^{\circledR}$ software on an interface desktop computer. Figure 13 shows the results of real-time PIL testing of the proposed adaptive DSMC with incorporated ADC uncertainties on control signals for the engine case study. The results verify that the adaptive DSMC is able to (i) remove the uncertainty in the model fast, (ii) track all the desired trajectories under ADC uncertainties, and (iii) operate in real-time since it is computationally efficient.

(a)
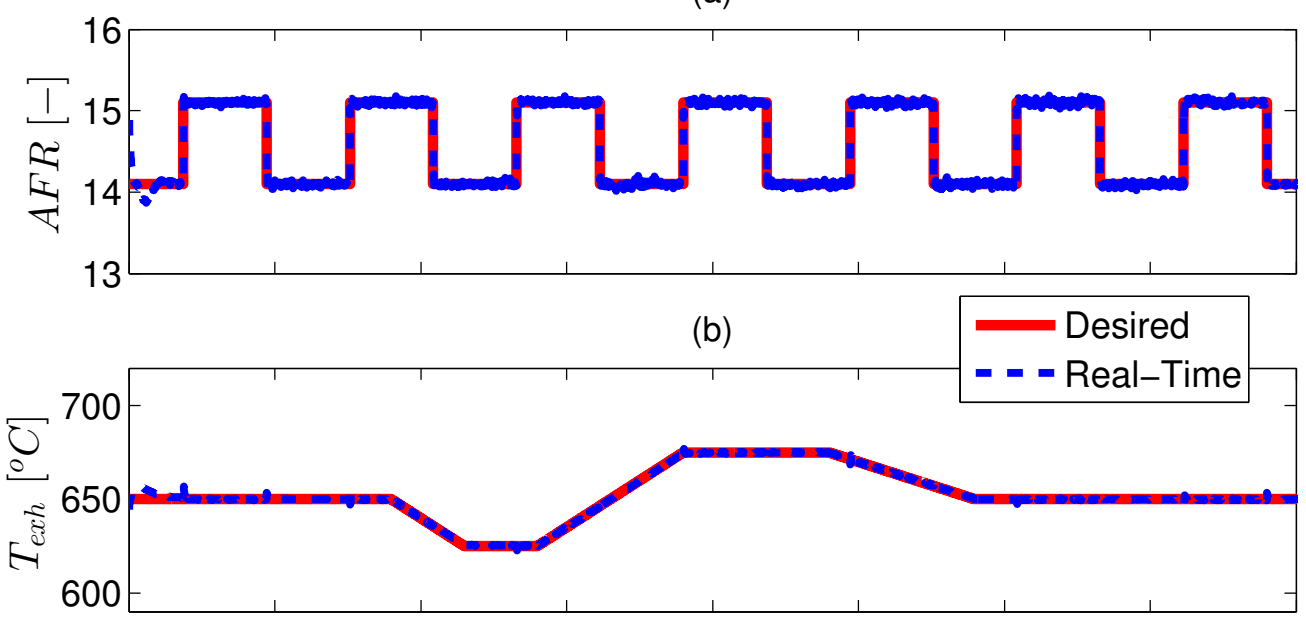

(c)

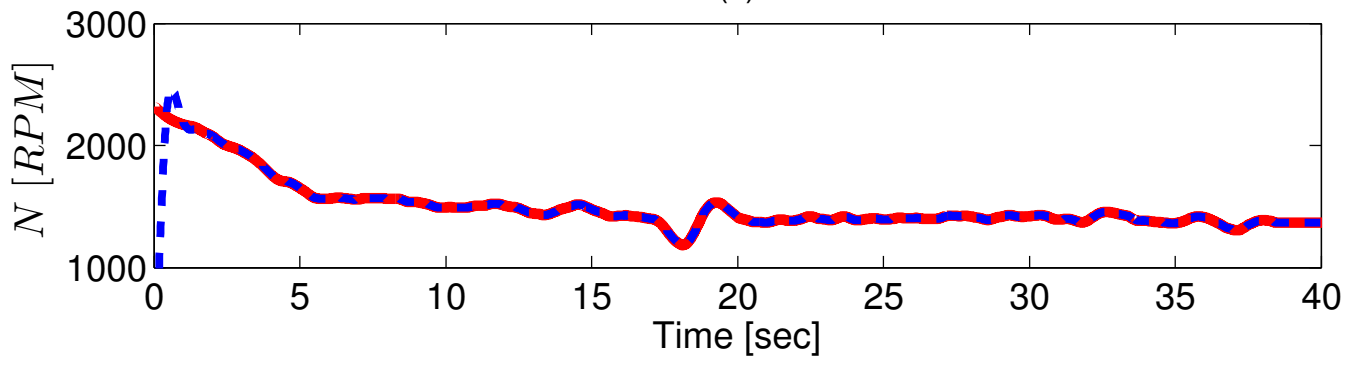

Figure 13: Real-time verification results of the adaptive robust DSMC for the engine control case study (10 $\mathrm{ms}$ sampling time and 10-bit quantization level).

\section{Summary and Conclusion}

A novel adaptive discrete sliding mode controller (DSMC) design for a general class of linear and nonlinear systems was proposed in this paper. 
The proposed controller design aspires to bridge the gap between the designed and implemented controllers with focus to overcome hardware (ADC) and model uncertainties. The ADC imprecisions were handled via an online uncertainty prediction and propagation mechanism. Next, the DSMC design was examined under multiplicative and additive types of model uncertainties. It was shown that the controller performance is sensitive to these uncertainties in the modeled dynamics. A discrete Lyapunov argument was employed to design adaptation laws to compensate for the unknown uncertainty terms in the controller structure. The final controller is an adaptive robust DSMC with incorporated ADC imprecisions which can handle both model and implementation uncertainties.

A processor-in-the-loop (PIL) setup was designed and implemented to verify the real-time performance of the proposed DSMC for a nonlinear automotive engine control problem. Real-time experimental and simulation results in this study show:

1. Real-Time Implementation: The ADC uncertainty prediction mechanism and the adaptation laws are computationally efficient for realtime operation. The proposed adaptation laws are able to quickly remove the errors in the modeled dynamics. Increasing the sampling time raises the required time for the adaptation laws to remove the errors in the model; however, these errors can be removed permanently. For the engine case study, all the additive and multiplicative unknown parameters were resolved within $2 \mathrm{sec}$ (sampling time=10 $\mathrm{ms}$ ).

2. Robustness to ADC Imprecisions: For the engine control problem, when the sampling time is increased from $10 \mathrm{~ms}$ to $35 \mathrm{~ms}$, the tracking errors from the baseline adaptive DSMC increase by 5-10 times. Additionally, by changing the ADC quantization level from 10 bit to 6 bit, the tracking errors of the baseline controller rise by up to 10 times. Incorporating the predicted ADC imprecisions into the adaptive DSMC improves the tracking errors by $50-60 \%$ compared to the baseline controller.

3. Robustness to Model Uncertainties: Four multiplicative and four additive unknown parameters within the engine control problem were considered to represent any mismatches in the modeled plant's dynamics. Each of these unknown parameters can cause up to $25 \%$ deviation from the nominal model in the corresponding dynamics. Although the baseline DSMC is too sensitive to any errors in the model and fails un- 
der these mismatches, the proposed adaptation technique provides an effective tool for permanent removal of the the errors due to unknown parameters, in less than $2 \mathrm{sec}$. The proposed adaptation laws were derived via the stability analysis of the closed loop system which not only remove the errors in the model, but also guarantees the asymptotic stability of the controller. The adaptive robust DSMC is able to enhance the trajectory tracking errors by more than $95 \%$, compared to the nonadaptive DSMC, and removes the errors in the engine dynamics by up to $98 \%$.

\section{Acknowledgement}

This material is based upon the work supported by the United States National Science Foundation under Grant No. 1434273. Prof. Rush Robinett III from Michigan Technological University, Dr. Ken Butts from Toyota Motor Engineering \& Manufacturing North America, and Adreas Hansen and Eric Choi from University of California, Berkeley are gratefully acknowledged for their technical comments during the course of this study. 


\section{References}

[1] J. B. Dabney, G. Barber, and D. Ohi. Estimating Direct Return on Investment of Independent Verification and Validation using COCOMOII. Proc. of the 10th IASTED Int. Conference on Software Engineering and Applications, 2006. Dallas, TX, USA.

[2] M. Shahbakhti, J. Li, and J. K. Hedrick. Early Model-Based Verification of Automotive Control System Implementation. 2012 American Control Conference, 2012. Montreal, Canada.

[3] K. Edelberg, M. Shahbakhti, and J. K. Hedrick. Incorporation of Implementation Impercesion in Automotive Control Design. 2013 American Control Conference, 2013. Washington, DC, USA.

[4] M. R. Amini, M. Shahbakhti, and A. Ghaffari. A Novel Singular Perturbation Technique for Model-based Control of Cold Start Hydrocarbon Emission. SAE Int. J. of Engines, 7(3):1290-1301, 2014.

[5] M. Shahbakhti, M.R. Amini, J. Li, S. Asami, and J.K. Hedrick. Early Model-Based Design and Verification of Automotive Control System Software Implementations. ASME J. of Dynamic System, Measurement, and Control, 137(2):021006, 2015.

[6] M. R. Amini, M. Shahbakhti, and J. K. Hedrick. Easily Verifiable Adaptive Sliding Mode Controller Design with Application to Automotive Engines. 2016. SAE Technical Paper 2016-01-0629.

[7] A. Hansen, M. Shahbakhti, and J. K. Hedrick. Impact of Implementation Impercision on Sliding Mode Controller Design for Automotive Cold Start Emissions. ASME 2012 Dynamic Systems and Control Conference.

[8] K. Edelberg, S. Pan, and J. K. Hedrick. A Discrete-Time Sliding Mode Formulation for Automotive Cold-Start Emission Control. 52nd IEEE Conference on Decision and Control, 2013. Florence, Italy.

[9] K. Edelberg, S. Pan, and J. K. Hedrick. Design of Automotive Control Systems Robust to Hardware Imprecision. ASME 2013 Dynamic Systems and Control Conference, 2013. Palo Alto, CA, USA. 
[10] M. R. Amini, M. Shahbakhti, and J. K. Hedrick. Discrete Sliding Controller Design with Robustness to Implementation Imprecisions via Online Uncertainty Prediction. 2016 American Control Conference, 2016. Boston, MA, USA.

[11] E. Misawa. Discrete-time sliding mode control for nonlinear systems with unmatched uncertainties and uncertain control vector. ASME J. of Dynamic System, Measurement, and Control, 119(3):503-512, 1997.

[12] J.-J.E. Slotine and L. Weiping. "Applied nonlinear control". Prenticehall Englewood Cliffs, NJ, Chapter 7 \& 8, 1991.

[13] V. Acary and B. Brogliato. Implicit Euler numerical scheme and chattering-free implementation of sliding mode systems. Systems $\&$ Control Letters, 59(5):284-293, 2010.

[14] C. Chan. Discrete adaptive sliding-mode tracking controller. Systems $\&$ Control Letters, 33(5):999-1002, 1997.

[15] S. Pan. Discrete Sliding Control for the Dynamics of Engine Cold Start. PhD thesis, UC Berkeley, 2014.

[16] D. Muñoz and D. Sbarbaro. An adaptive sliding-mode controller for discrete nonlinear systems. IEEE Transactions on Industrial Electronics, 47(3):574-581, 2000.

[17] Y. Fang, T. Chow, and X. Li. Use of a recurrent neural network in discrete sliding-mode control. IEEE Proceedings-Control Theory and Applications, 146(1):84-90, 1999.

[18] S. Pan and J. K. Hedrick. Tracking Controller Design for MIMO Nonlinear Systems With Application to Automotive Cold Start Emission Reduction. Journal of Dynamic Systems, Measurement, and Control, 137(10):101013, 2015.

[19] M. R. Amini, M. Shahbakhti, S. Pan, and J. K. Hedrick. Handling Model and Implementation Uncertainties via An Adaptive Discrete Sliding Mode Controller Design. ASME 2016 Dynamic Systems and Control Conference, 2016. Minneapolis, MN, USA (Accepted in May 2016). 
[20] S. Pan, K. Edelberg, and J. K. Hedrick. Discrete adaptive sliding control of automotive powertrains. 2014 American Control Conference, 2014. Portland, OR, USA.

[21] I. Barkana. Defending the Beauty of the Invariance Principle. International Journal of Control, 87(1):186-206, 2014.

[22] H. Khalil and J. Grizzle. "Nonlinear Systems", volume 3. Prentice-hall Englewood Cliffs, NJ, 1996.

[23] I. Barkana. The New Theorem of Stability-Direct Extension of Lyapunov Theorem. Mathematics in Engineering, Science 83 Aerospace (MESA), 6(3), 2015.

[24] J. Cook, J. Sun, J. H. Buckland, I. Kolmanovsky, H. Peng, and J. Grizzle. Automotive Powertrain Control, A Survey. Asian J. of Control, $8(3): 237-260,2006$.

[25] J. Cook, J. Sun, and J. Grizzle. Opportunities in Automotive Powertrain Control Applications. Proceedings of the IEEE 7th Conference on Control and Its Applications, 2002. Glasgow, UK.

[26] B. T. Shaw II. Modelling and Control of Automotive Coldstart Hydrocarbon Emissions. PhD thesis, UC Berkeley, 2002.

[27] P. R. Sanketi, J. C. Zavala, J. K. Hedrick, M. Wilcutts, and T. Kaga. A Simplified Catalytic Converter Model for Automotive Coldstart Applications with Adaptive Parameter Fitting. 8th Int. Symposium on Advanced Vehicle Control, 2006. Taipei, Taiwan.

[28] P. R. Sanketi. Coldstart Modeling and Optimal Control Design for Automotive SI Engines. PhD thesis, UC Berkeley, 2009.

[29] R. Salehi, M. Shahbakhti, and J. K. Hedrick. Real-time hybrid switching control of automotive cold start hydrocarbon emission. ASME J. of Dynamic System, Measurement, and Control, 136(4):041002, 2014.

[30] M. Razmara, M. Bidarvatan, M. Shahbakhti, and R. Robinett III. Novel Exergy-Wise Predictive Control of Internal Combustion Engines. 2016 American Control Conference, 2016. Boston, MA, USA. 
[31] M. Razmara, M. Bidarvatan, M. Shahbakhti, and R. Robinett III. Innovative Exergy-Based Combustion Phasing Control of IC Engines. 2016. SAE Technical Paper 2016-01-0815.

[32] D. Efimov, A. Polyakov, L. Fridman, W. Perruquetti, and J. Richard. Delayed Sliding Mode Control. Automatica, 64:37-43, 2016.

[33] X. Zhang, H. Su, and R. Lu. Second-Order Integral Sliding Mode Control for Uncertain Systems with Control Input Time Delay Based on Singular Perturbation Approach. IEEE Transactions on Automatic Control, 60(11):3095-3100, 2015.

[34] P. Yang, J. Ni, X. Pan, and J. Liu. Global Robust Sliding Mode Control for Time-Delay Systems with Mismatched Uncertainties. Circuits, Systems, and Signal Processing, pages 1-12, 2015.

\section{Nomenclature}

\begin{tabular}{ll} 
Parameters/ & \\
Variables & Description \\
\hline$A F R$ & air fuel ratio, $[-]$ \\
$J$ & effective engine inertia, $\left[\mathrm{kg} \cdot \mathrm{m}^{2}\right]$ \\
$m_{a}$ & air mass inside the intake manifold, $[\mathrm{kg}]$ \\
$\dot{m}_{f}$ & fuel mass flow into the cylinder, $[\mathrm{kg} / \mathrm{s}]$ \\
$\dot{m}_{a i}$ & air mass flow into the intake manifold, $[\mathrm{kg} / \mathrm{s}]$ \\
$\dot{m}_{a o}$ & air mass flow into the cylinders, $[\mathrm{kg} / \mathrm{s}]$ \\
$\dot{m}_{f c}$ & commanded fuel mass flow, $[\mathrm{kg} / \mathrm{s}]$ \\
$T_{e x h}$ & exhaust gas temperature, $[\mathrm{K}]$ \\
$\Delta$ & spark timing, $\left[{ }^{o}\right.$ ATDC] \\
$\omega_{e}$ & engine speed, $[\mathrm{rad} / \mathrm{s}]$ \\
$N$ & engine speed, $[R P M]$ \\
$\tau_{f}$ & fuel evaporation time constant, $[\mathrm{s}]$ \\
$\tau_{e}$ & exhaust gas time constant, $[\mathrm{s}]$ \\
$T$ & sampling time, $[\mathrm{s}]$ \\
$\rho$ & tunable DSMC gain, $[-]$ \\
$\rho_{\beta}$ & adaptation gain for multiplicative uncertainty, $[-]$ \\
$\rho_{\alpha}$ & adaptation gain for additive uncertainty, $[-]$ \\
\hline
\end{tabular}




\section{Appendix}

- Engine Plant Model Functions:

$$
\begin{gathered}
A F I=\cos (0.13(\text { AFR }-13.5)) \\
T_{E}=30000 m_{a}-0.4 \omega_{e}-100 \\
\tau_{e}=2 \pi / \omega_{e} \\
\dot{m}_{a o}=0.0254 m_{a} \cdot \omega_{e} \cdot \eta_{v o l} \\
\eta_{v o l}=m_{a}^{2}\left(-0.1636 \omega_{e}^{2}-7.093 \omega_{e}-1750\right) \\
+m_{a}\left(0.0029 \omega_{e}^{2}-0.4033 \omega_{e}+85.38\right) \\
-\left(1.06 e-5 \omega_{e}^{2}-0.0021 \omega_{e}-0.2719\right)
\end{gathered}
$$

- Engine Plant Model Constants:

\begin{tabular}{ll}
\hline \hline Constant & Value [unit] \\
\hline$J$ & $0.1454\left[\mathrm{~m}^{2} \mathrm{~kg}\right]$ \\
$\tau_{f}$ & $0.06[1 / \mathrm{sec}]$ \\
\hline \hline
\end{tabular}

